Article

\title{
Design, Synthesis and Docking Studies of Novel Macrocyclic Pentapeptides as Anticancer Multi-Targeted Kinase Inhibitors
}

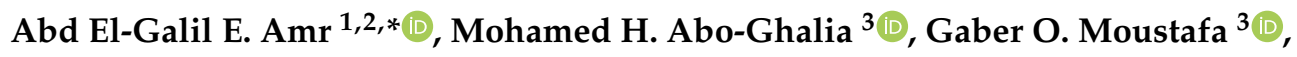 \\ Mohamed A. Al-Omar ${ }^{1}$ (D), Eman S. Nossier ${ }^{4}$ and Elsayed A. Elsayed ${ }^{5,6}$ (D) \\ 1 Pharmaceutical Chemistry Department, Drug Exploration \& Development Chair (DEDC), \\ College of Pharmacy, King Saud University, Riyadh 11451, Saudi Arabia; malomar1@ksu.edu.sa \\ 2 Applied Organic Chemistry Department, National Research Centre, 12622 Dokki, Giza, Egypt \\ 3 Department of Peptide Chemistry, National Research Centre, 12622 Dokki, Giza, Egypt; \\ mhaboghalia@yahoo.com (M.H.A.-G.); gosman79@gmail.com (G.O.M.) \\ 4 Department of Pharmaceutical Chemistry, Faculty of Pharmacy (Girls), Al-Azhar University, 11754 Cairo, \\ Egypt; dr.emannossier@gmail.com \\ 5 Bioproducts Research Department, Zoology Department, Faculty of Science, King Saud University, \\ Riyadh 11451, Saudi Arabia; eaelsayed@ksu.edu.sa \\ 6 Chemistry of Natural and Microbial Products Department, National Research Centre, 12622 Dokki, \\ Cairo, Egypt \\ * Correspondence: aeamr1963@yahoo.com; Tel.: +966-565-148-750
}

Received: 5 September 2018; Accepted: 18 September 2018; Published: 20 September 2018

\begin{abstract}
A series of macrocyclic pyrido-pentapeptide candidates 2-6 were synthesized by using $\mathrm{N}, \mathrm{N}$-bis-[1-carboxy-2-(benzyl)]-2,6-(diaminocarbonyl)pyridine $\mathbf{1 a}, \mathbf{b}$ as starting material. Structures of the newly synthesized compounds were established by IR, ${ }^{1} \mathrm{H}$ and ${ }^{13} \mathrm{C}-\mathrm{NMR}$, and MS spectral data and elemental analysis. The in-vitro cytotoxicity activity was investigated for all compounds against MCF-7 and HepG-2 cell lines and the majority of the compounds showed potent anticancer activity against the tested cell lines in comparison with the reference drugs. Out of the macrocyclic pyrido-pentapeptide based compounds, $5 \mathrm{c}$ showed encouraging inhibitory activity on MCF-7 and HepG-2 cell lines with $\mathrm{IC}_{50}$ values $9.41 \pm 1.25$ and $7.53 \pm 1.33 \mu \mathrm{M}$, respectively. Interestingly, $\mathbf{5 c}$ also demonstrated multitarget profile and excellent inhibitory activity towards VEGFR-2, CDK-2 and PDGFR $\beta$ kinases. Furthermore, molecular modeling studies of the compound $\mathbf{5 c}$ revealed its possible binding modes into the active sites of those kinases.
\end{abstract}

Keywords: macrocyclic pentapeptides; in vitro anticancer activity; multitarget; molecular modeling studies

\section{Introduction}

Cancer is still considered one of the most serious diseases threatening human life. In the past three decades, there enormous efforts have been undertaken to confront cancer diseases. Within such efforts, many therapeutic agents have been developed to treat cancer patients in their early, as well as late cancer-developing stages [1]. These agents vary from antibiotics, chemically synthesized compounds, and natural products-based drugs.

Macrocyclic motifs are commonly defined as a ring system containing 12 or more atoms [2]. They are privileged scaffolds in the fields of chemistry, biology, and medicine [3-5]. There are different classes of macrocycles like peptidic and nonpeptidic natural products, non-natural (synthetic) peptides and non-natural (synthetic) macrocycles [6]. 
Furthermore, peptides constitute a major class of important anticancer therapeutic agents [7]. Chemically synthesized peptides have been reported to exhibit antimicrobial, anti-inflammatory [8-16], as well as anticancer properties [17-21]. We have previously explored the analytical and biological characteristics of some bis-amino acid and peptide conjugates of dipicolinic acid [22]. Our studies of these compounds exemplified by compound [A] (Figure 1) revealed an interesting anticancer activity, probably via DNA intercalation, as well as an outstanding metal sensor property, particularly, for pollutant lead $\left(\mathrm{Pb}^{2+}\right)$ cations [23].<smiles>CCCC(NC(=O)C(C)C)C(=O)NC(CCC)C(=O)NCCCCC(NC(=O)C(CC(C)C)NC(=O)c1cccc(C(=O)NC(CC(C)C)C(=O)NC(CCC)C(=O)OC)n1)C(=O)OC</smiles>

Figure 1. Structure of the anticancer agent.

The advances in molecular biology and genetics help in identification of molecular targets that are related to cancer cells or overexpressed on them. The design of compounds affecting these targets improves the development of more selective anticancer drugs with less toxic side effects [24]. Macrocycles were reported to display antitumor properties, which may be attributed to inhibition of different enzymes involved in carcinogenesis cases. The prominent examples of macrocycles, illustrated in Figure 2, exhibited a potent inhibitory activity against various kinases, e.g., CDK-2, VEGFR-2, JAK-2, FLT-3, PDK-1 and EGFR [6,25-28].<smiles>O=S(=O)(NCCCNc1nc(Nc2cccc(Br)c2)ncc1Br)c1ccccc1</smiles>

B (CDK-2 \&VEGFR-2 inhibitor)<smiles>CN(C)C/C=C/CCOc1cccc(-c2nc(Nc3cccc(CN(C)C)c3)nc3[nH]cnc23)c1</smiles>

C (PDK-1, CDK-2, Flt-3 \& JAK-2 inhibitor)<smiles>CN(C)C/C=C\CCOc1cccc(-c2ccnc(Nc3cccc(CN(C)C)c3)n2)c1</smiles>

SG1317/TG02 (CDK-2, JAK-2 \& FLT-3 inhibitor)

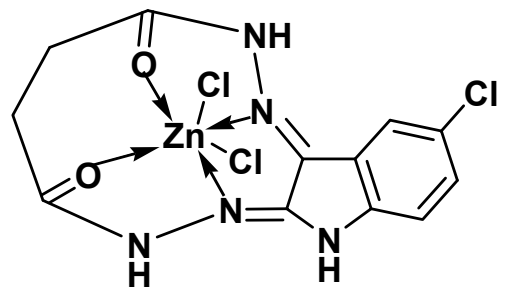

D (EGFR inhibitor)

Figure 2. Reported and proposed macrocyclic conjugates with anticancer and different kinases inhibitory activity. 
In view of these observations and as continuation of our previous works in peptide heterocyclic chemistry, we have herein synthesized some new linear and macrocyclic peptidopyridine derivatives, and tested their anticancer activity in comparison to Tamoxifen and 5-Fluorouracil ${ }^{\circledR}$ as positive controls. Furthermore, they were screened for their inhibitory activity against VEGFR-2, EGFR, PDGFR $\beta$ and CDK-2 enzymes. Additionally, molecular modeling study was performed to explore the most appropriate binding modes of the most potent target compounds.

\section{Results and Discussion}

\subsection{Chemistry}

In the previous work $[9,29]$, L-amino acid methyl esters was initially coupled with dipicolinic acid via the conventional acid chloride method to give the corresponding 2,6-bis- $\mathrm{N}^{\alpha}$-L-diamino acid pyridine methyl ester derivatives. In the present work, a series of linear and macrocyclic pyridopentapeptide derivatives 2-6 were synthesized based on N,N-bis-[1-carboxy-2-(benzyl)]-2,6-

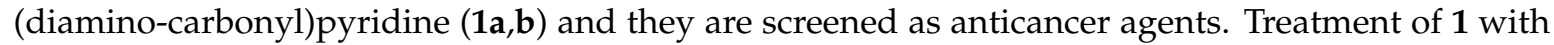
L-amino acid methyl ester hydrochloride in the presence of ethyl chloroformate in dichloromethane afforded the corresponding tetrapeptide pyridine methyl ester derivatives $\mathbf{2 a - c}$, respectively (Scheme 1).<smiles>[R1]C(N)C(=O)OC</smiles><smiles>[R]C(NC(=O)c1cccc(C(=O)NC([R])C(=O)OC)n1)C(=O)OC</smiles><smiles>[R]C(NC(=O)c1cccc(C(=O)NC([R])C(=O)NC([R2])C(=O)OC)n1)C(=O)NC([R])C(=O)OC</smiles>

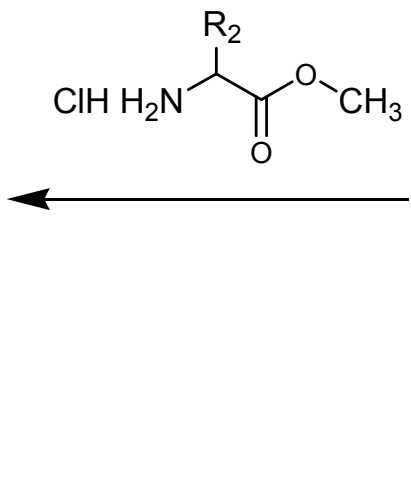

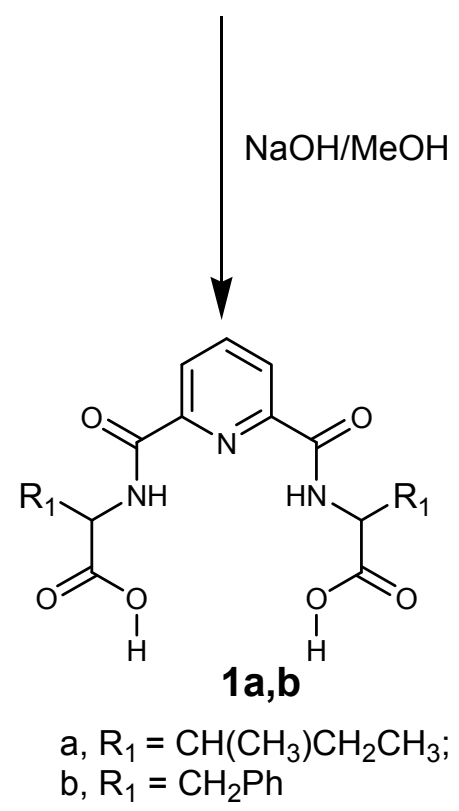

a, $\mathrm{R}_{1}=\mathrm{R}_{2}=\mathrm{CH}\left(\mathrm{CH}_{3} \mathrm{CH}_{2} \mathrm{CH}_{3}\right.$;

b, $\mathrm{R}_{1}=\mathrm{CH}\left(\mathrm{CH}_{3}\right) \mathrm{CH}_{2} \mathrm{CH}_{3} ; \mathrm{R}_{2}=\mathrm{CH}_{2} \mathrm{Ph}$

c, R1 $=\mathrm{CH}_{2} \mathrm{Ph} ; \mathrm{R}_{2}=\mathrm{CH}\left(\mathrm{CH}_{3}\right) \mathrm{CH}_{2} \mathrm{CH}_{3}$

Scheme 1. Synthetic pathway for compounds $2 \mathbf{a}-\mathbf{c}$. 
Hydrolysis of $\mathbf{2 a - c}$ with methanolic sodium hydroxide to afford the corresponding tetrapeptide pyridine derivatives $3 \mathbf{a}-\mathbf{c}$, which were cyclized with L-lysine methyl ester by different methods to afford the corresponding cyclic pentapeptide esters $\mathbf{4 a - c}$, respectively. The cyclized pentapeptide esters 4a-c was hydrolyzed with methanolic sodium hydroxide to give the corresponding cyclic pentapeptide acids $5 \mathbf{a}-\mathbf{c}$, or by hydrazonolysis with hydrazine hydrate in methanol to give the corresponding cyclic pentapeptide acid hydrazides $\mathbf{6 a}-\mathbf{c}$, respectively (Scheme 2).

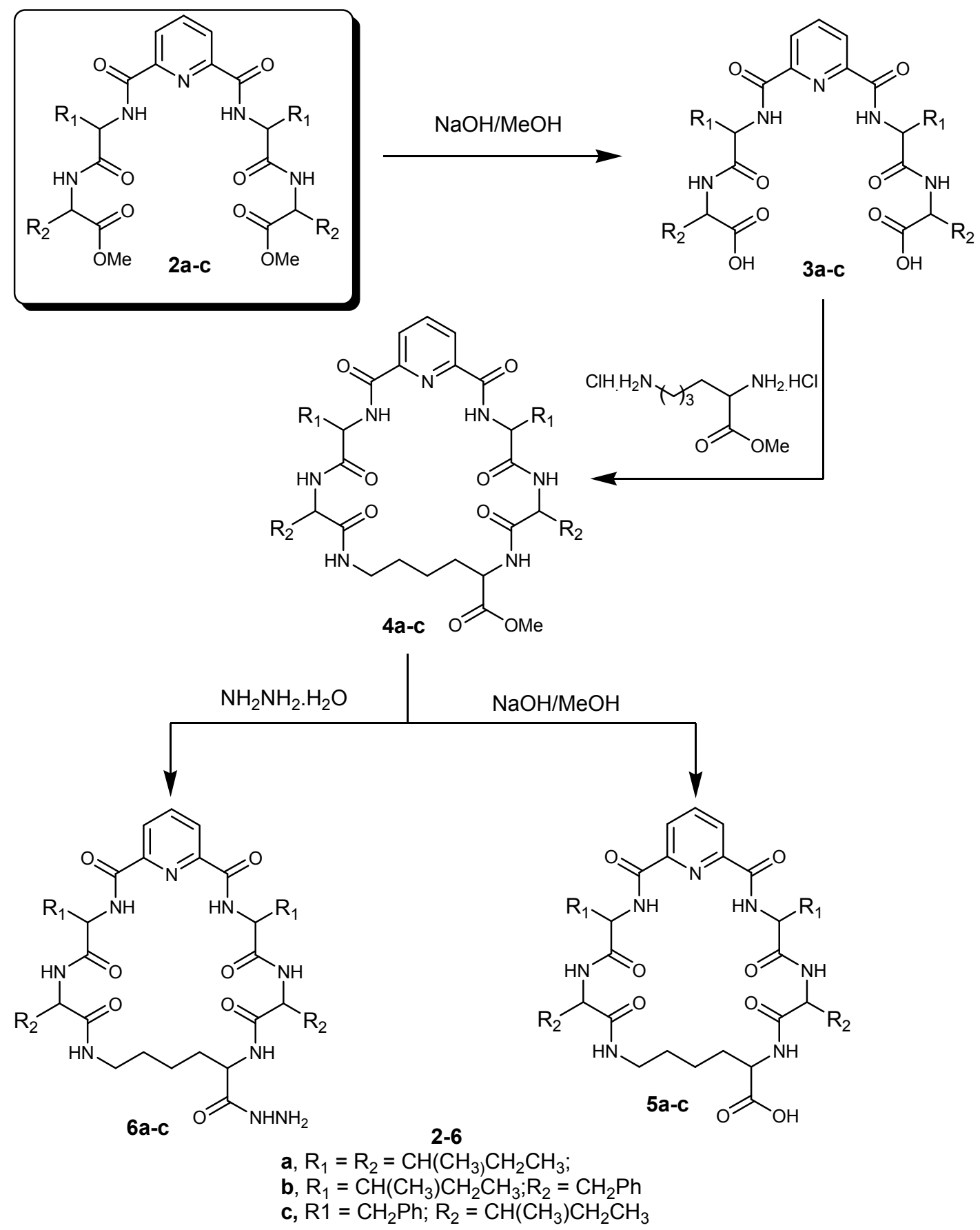

Scheme 2. Synthetic pathway for compounds 3-6. 


\subsection{Anticancer Activity}

The cytotoxic effects of all newly synthesized compounds were evaluated against MCF-7 and HepG-2 cell lines. Results obtained (Figure 3) showed that all prepared compounds affected both cell lines in a dose-dependent manner, where increasing the applied concentration gradually decreased cell viability. All tested compounds exhibited moderate to excellent cytotoxic activities on both tested cell lines in comparison with control drugs. Compounds $\mathbf{4 a}, \mathbf{4 b}, \mathbf{5 a}, \mathbf{5} \mathbf{b}, \mathbf{6} \mathbf{a}$ and $\mathbf{6} \mathbf{b}$ were more potent at lower range of tested concentrations. However, compounds $\mathbf{2} \mathbf{c}, \mathbf{3 a}, \mathbf{4 a}$ and $\mathbf{6} \mathbf{c}$ were considered inactive against MCF-7 cells, since they showed no practical $\mathrm{IC}_{50}(>100 \mu \mathrm{M})$. Concerning $\mathrm{IC}_{50}$ data (Table 1), it was noticed that only compounds $\mathbf{6 a}$ and $\mathbf{6 b}$ affected MCF-7 cells more than HepG-2 cells $\left(\mathrm{IC}_{50}=11.83 \pm 1.62\right.$ and $10.87 \pm 1.10 \mu \mathrm{M}$ for MCF-7, and $12.44 \pm 1.3$ and $11.53 \pm 1.70 \mu \mathrm{M}$ for HepG-2, respectively). While, the rest of the prepared compounds showed more noticeable anticancer activities in case of HepG-2 cells rather than MCF-7 cells. Comparing the obtained $\mathrm{IC}_{50}$ values for HepG-2 cells with those of the control drugs explored that compound $5 \mathrm{c}$ was the most potent in comparison with tamoxifen and 5-fluorouracil ( $\mathrm{IC}_{50}=7.53 \pm 1.33,29.38 \pm 1.15$ and $43.84 \pm 1.84 \mu \mathrm{M}$, respectively). Compounds $\mathbf{2 a}, \mathbf{2 b}, \mathbf{3 b}, \mathbf{4 a}-\mathbf{c}, \mathbf{5 a}-\mathbf{c}$ and $\mathbf{6 a}-\mathbf{c}$ afforded higher cytotoxic activity, while compounds $\mathbf{3 a}$ and $3 c$ gave approximately equipotent cytotoxic activity $\left(\mathrm{IC}_{50}=26.01 \pm 2.35\right.$ and $26.64 \pm 1.85 \mu \mathrm{M}$, respectively). For MCF-7 cell lines, $5 \mathrm{c}$ was also the most active derivative in comparison with tamoxifen $\left(\mathrm{IC}_{50}=9.41 \pm 1.25\right.$ and $22.40 \pm 2.42 \mu \mathrm{M}$, respectively), followed by compounds $4 \mathbf{b}, \mathbf{6 b}, \mathbf{5 b}, \mathbf{6} \mathbf{a}$ and $\mathbf{6 a}$ $\left(\mathrm{IC}_{50}=10.45 \pm 1.33,10.87 \pm 1.10,11.32 \pm 1.15,11.83 \pm 1.62\right.$ and $12.67 \pm 2.40 \mu \mathrm{M}$, respectively $)$
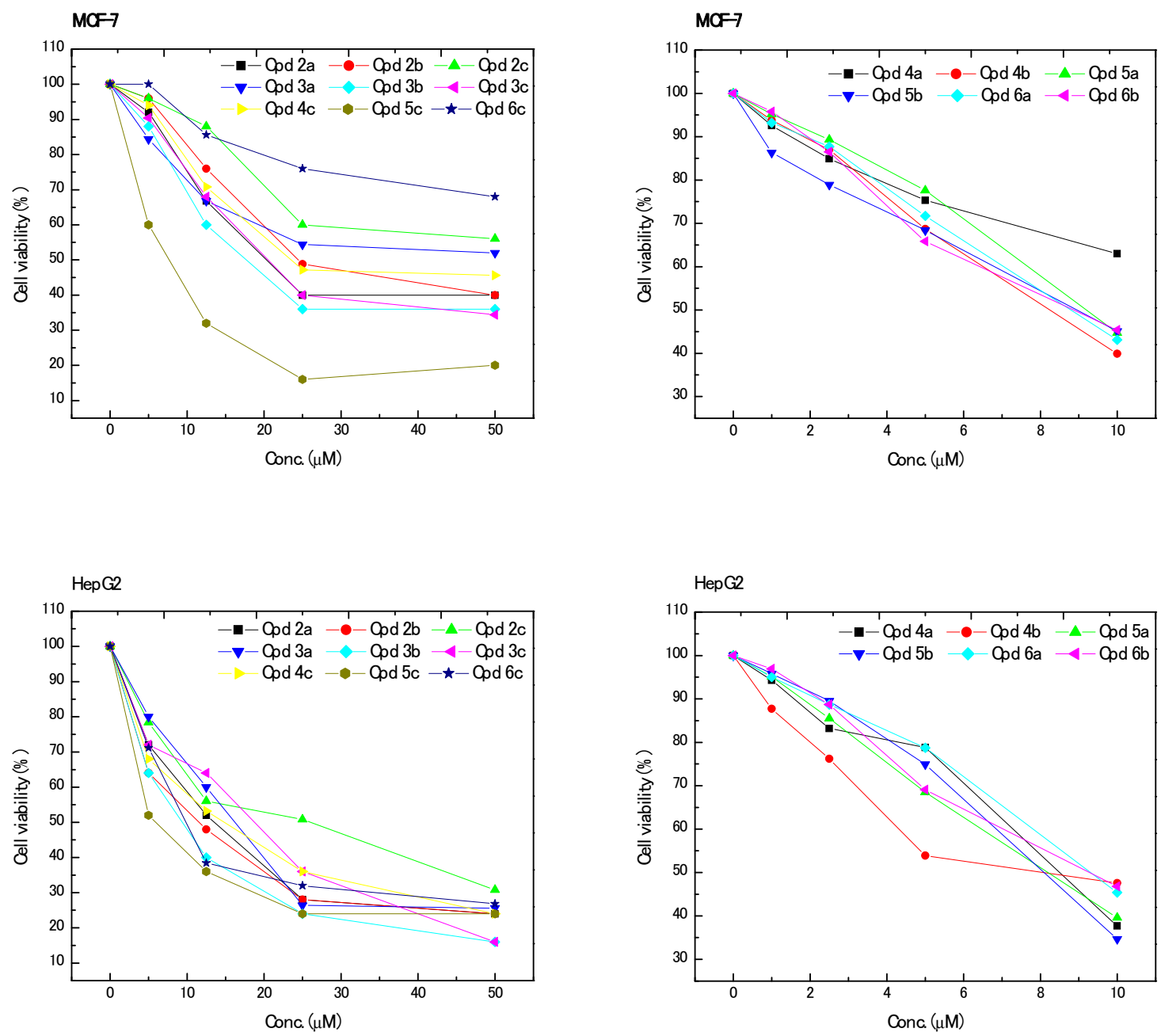

Figure 3. Effect of different concentrations of the prepared compounds on the viability of HepG-2 and MCF-7 cell lines. Data are expressed as means \pm SEM (standard error mean). 
Table 1. $\mathrm{IC}_{50}$ of the tested compounds 2-6 against MCF-7 and HepG-2 cell lines.

\begin{tabular}{|c|c|c|}
\hline \multirow{2}{*}{ Compound } & \multicolumn{2}{|c|}{$\mathrm{IC}_{50}($ Mean \pm SEM $)(\mu \mathrm{M})$} \\
\hline & MCF-7 & HepG-2 \\
\hline $2 a$ & $31.64 \pm 1.30$ & $20.37 \pm 1.36$ \\
\hline $2 b$ & $32.58 \pm 1.50$ & $15.80 \pm 1.66$ \\
\hline $2 c$ & - & $35.52 \pm 1.83$ \\
\hline $3 a$ & - & $26.01 \pm 2.35$ \\
\hline $3 b$ & $25.33 \pm 1.18$ & $13.54 \pm 1.45$ \\
\hline $3 c$ & $29.55 \pm 2.06$ & $26.64 \pm 1.85$ \\
\hline $4 a$ & - & $11.59 \pm 2.70$ \\
\hline $4 b$ & $10.45 \pm 1.33$ & $10.25 \pm 2.20$ \\
\hline $4 c$ & $29.15 \pm 1.39$ & $18.84 \pm 1.47$ \\
\hline $5 a$ & $12.67 \pm 2.40$ & $11.19 \pm 1.95$ \\
\hline $5 b$ & $11.32 \pm 1.15$ & $10.09 \pm 2.05$ \\
\hline $5 c$ & $9.41 \pm 1.25$ & $7.53 \pm 1.33$ \\
\hline $6 a$ & $11.83 \pm 1.62$ & $12.44 \pm 1.3$ \\
\hline $6 b$ & $10.87 \pm 1.10$ & $11.53 \pm 1.70$ \\
\hline $6 c$ & - & $12.07 \pm 1.68$ \\
\hline Tamoxifen & $22.40 \pm 2.42$ & $29.38 \pm 1.15$ \\
\hline 5-Fluorouracil ${ }^{\circledR}$ & - & $43.84 \pm 1.84$ \\
\hline
\end{tabular}

$\mathrm{IC}_{50}$ : Compound concentration required to inhibit the cell viability by $50 \%, \mathrm{SEM}=$ standard error mean; each value is the mean of three values.

By analysis of the previous results, the cytotoxic activities of the tested compounds, with the exception of compound $4 c$, were in the following order compound $5>6>4>3>2$. Concerning the structure-activity relationships of the synthesized compounds, it was observed that the open chain derivatives $\mathbf{2}$ and $\mathbf{3}$ have lower activity than the cyclized derivatives 4-6. Furthermore, insertion of benzyl moiety at $\mathrm{R}_{1}$ adjacent to the pyridine scaffold in $2 \mathrm{c}$ and $3 c$ gave a marked decrease in the cytotoxic activity compared to their series $\mathbf{2} \mathbf{a}, \mathbf{b}$ and $\mathbf{3} \mathbf{a}, \mathbf{b}$, respectively. This decrease may be due to formation of steric hindrance. The enhanced anticancer activities of compounds 4-6 may be contributed to the cyclization of these compounds. Contrary to what was found in the open chain derivatives $2 \mathrm{c}$ and $3 \mathbf{c}$, the increased aromaticity of the cyclopeptide by the location of the phenyl group of phenylalanine neighboring the pyridine nucleus may be the cause of improved activity of compound $5 c>5 b>5 a$.

\subsection{In Vitro Enzymatic Assays}

Upon cellular screening on MCF-7 and HepG-2, compound 5c exhibited higher anticancer activity in comparison with tamoxifen and 5-fluorouracil. So, it was subjected for in vitro inhibition assessment against a panel of four different kinases; VEGFR-2, EGFR, PDGFR $\beta$ and CDK-2 kinases using staurosporine as multitarget inhibitor. As shown in Table 2 and Figure 4, compound 5c exhibited three folds increase in the inhibitory effect against VEGFR-2 $\left(\mathrm{IC}_{50}=0.01 \pm 1.25 \mu \mathrm{M}\right)$, two folds increase against $\mathrm{CDK}-2\left(\mathrm{IC}_{50}=0.06 \pm 1.27 \mu \mathrm{M}\right)$ and approximately equipotent against PDGFR $\beta\left(\mathrm{IC}_{50}=0.08 \pm 1.45 \mu \mathrm{M}\right)$ in comparison with staurosporine $\left(\mathrm{IC}_{50}=0.03 \pm 1.10,0.11 \pm 1.13\right.$ and $0.07 \pm 1.65 \mu \mathrm{M}$, respectively). Furthermore, the inhibitory activity of compound $\mathbf{5 c}$ was moderate against EGFR in comparison with the reference $\left(\mathrm{IC}_{50}=0.14 \pm 1.00\right.$ and $0.02 \pm 1.32 \mu \mathrm{M}$, respectively). These data suggested that compound $\mathbf{5 c}$ is a promising multitarget kinase inhibitor. 
Table 2. Inhibitory evaluation of compound $5 c$ against VEGFR-2, EGFR, PDGFR $\beta$ and CDK-2 kinases.

\begin{tabular}{ccc}
\hline \multirow{2}{*}{ Kinase } & \multicolumn{2}{c}{ IC $_{\mathbf{5 0}}$ (Mean \pm SEM) $(\boldsymbol{\mu M})$} \\
\cline { 2 - 3 } & $\mathbf{5}$ & Staurosporine \\
\hline VEGFR-2 & $0.01 \pm 1.25$ & $0.03 \pm 1.10$ \\
EGFR & $0.14 \pm 1.00$ & $0.02 \pm 1.32$ \\
PDGFR $\beta$ & $0.08 \pm 1.45$ & $0.07 \pm 1.65$ \\
CDK-2 & $0.06 \pm 1.27$ & $0.11 \pm 1.13$ \\
\hline
\end{tabular}

$\mathrm{IC}_{50}$ : Compound concentration required to inhibit the enzyme activity by $50 \%, \mathrm{SEM}=$ Standard error mean; each value is the mean of three values.

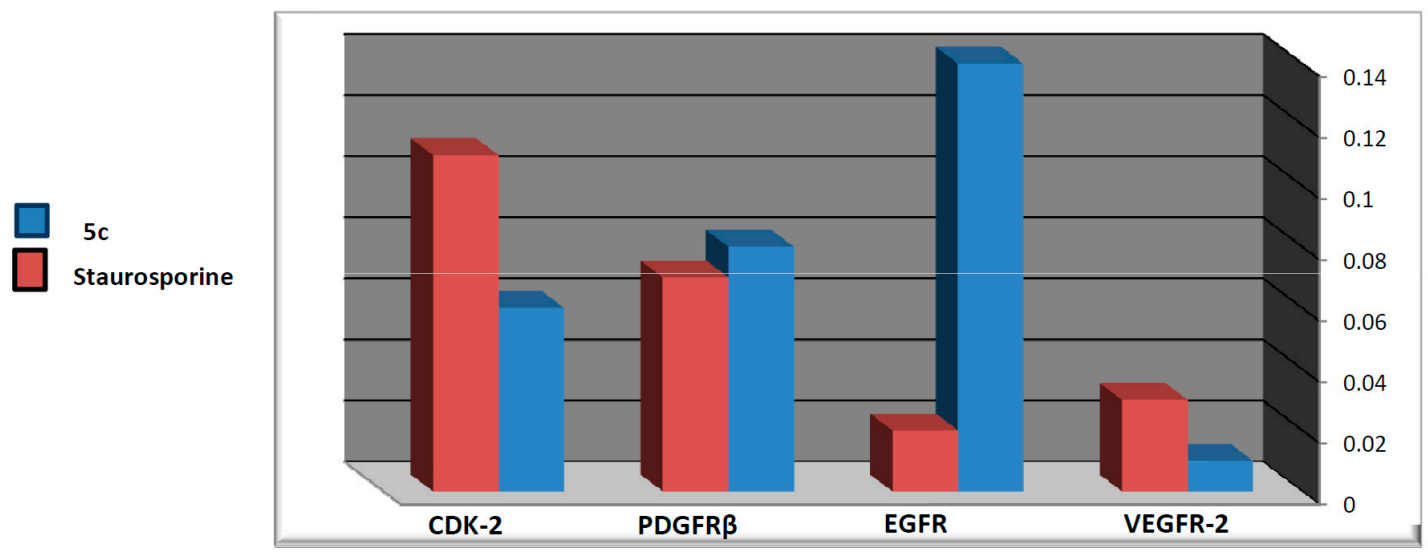

Figure 4. $\mathrm{IC}_{50}$ of compound $5 \mathrm{c}$ against VEGFR-2, EGFR, PDGFR $\beta$ and CDK-2 kinases in comparison with staurosporine.

\subsection{Molecular Modeling Studies}

The kinase inhibitory assay revealed that compound $5 \mathrm{c}$ showed promising inhibitory activity against three kinases, namely VEGFR-2, CDK-2 and PDGFR $\beta$. So, docking simulation was performed using Molecular Operating Environment $\left(\mathrm{MOE}^{\circledR}\right) 2008.10$ [30,31] to predict the binding modes, affinities, and orientations of compound $5 \mathbf{a}$ at the active sites of them. The X-ray crystallographic structure of PDGFR $\beta$ was not fully resolved [32]. On the other hand, the X-ray crystallographic structures were reported for VEGFR-2 (pdb code: 4ASD) [33] with sorafenib and for CDK-2 (PDB ID: 2J9M) [34] with PY8. So the docking study was achieved for both VEGFR-2 and CDK-2 kinases

The binding model, shown in Figure 5, was exemplified by the interaction of compound $\mathbf{5 c}$ with VEGFR-2. The carboxylic moiety of $\mathbf{5 c}$ shared in the binding pattern with three hydrogen bond donors; one H-bond was between CO group and the backbone of His1026 (distance: $2.99 \AA$ ), and the others were between $\mathrm{OH}$ group and the sidechain of Asp1046 (distance: 1.92 and 2.65 $\AA$ ). Moreover, the residues Ser884, Arg1027 and Leu1049 were inserted nicely inside the centre of cyclic pentapeptide scaffold. 


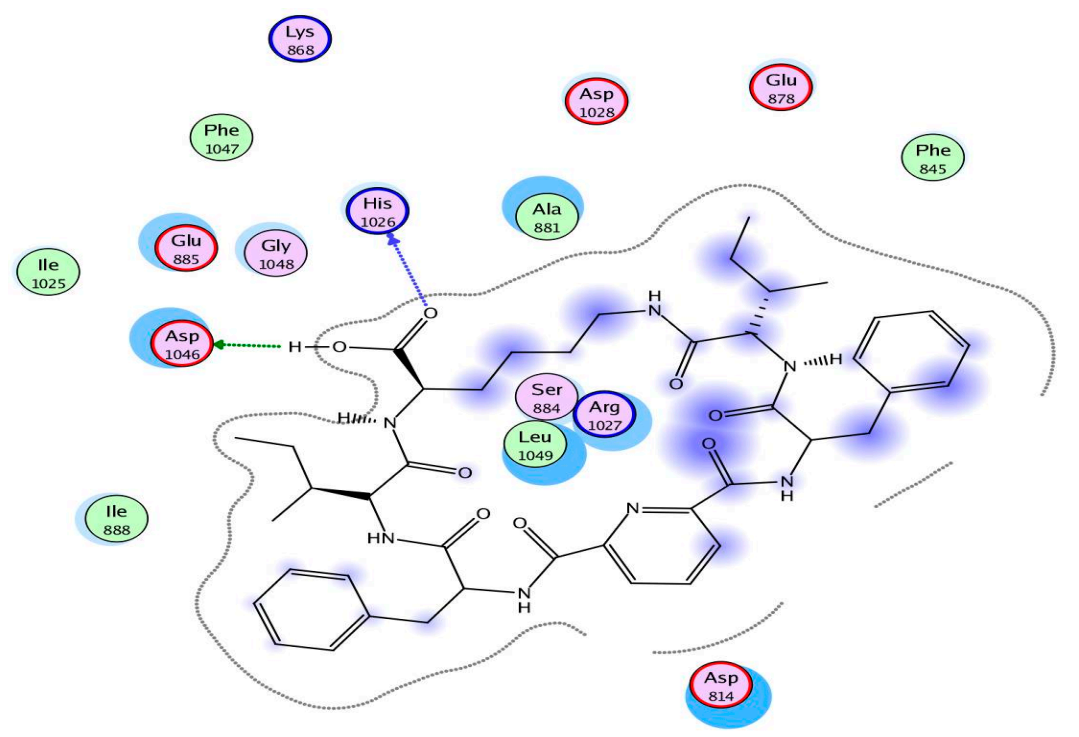

(A)

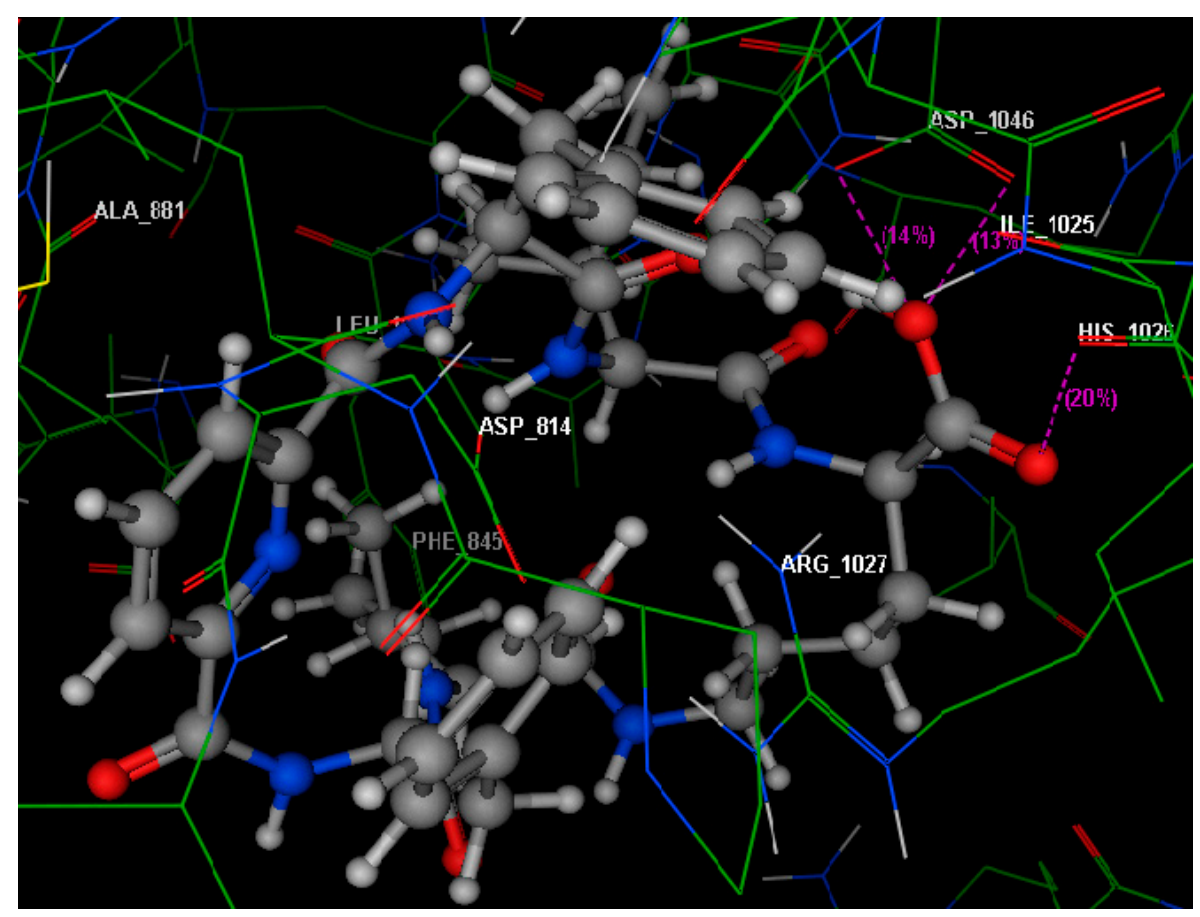

(B)

Figure 5. Docking solution for $5 \mathbf{c}$ in the active site of VEGFR-2; $(\mathbf{A}, \mathbf{B})$ showing $2 \mathrm{D}$ and 3D ligand-receptor interactions (hydrogen bonds are illustrated as arrows; $\mathrm{C}$ atoms are colored gray, $\mathrm{N}$ blue and $\mathrm{O}$ red).

Interaction of compound $\mathbf{5 c}$ with the binding site of CDK-2 kinase was illustrated in Figure 6. There was H-bond acceptor between $\mathrm{CO}$ of the carboxylic group and the backbone of Asp86 (distance: $2.89 \AA$ ). Another H-bond acceptor was established between the amide nitrogen and the backbone of Ile10 (distance: $1.80 \AA$ ). Furthermore, carbonyl group placed at p-2 of pyridine ring was linked to backbone of Glu12 (distance: $2.66 \AA$ ). In addition, Lys89 located in the centre of cyclic pentapeptide scaffold formed arene-cation interaction with centroid of benzyl ring. 


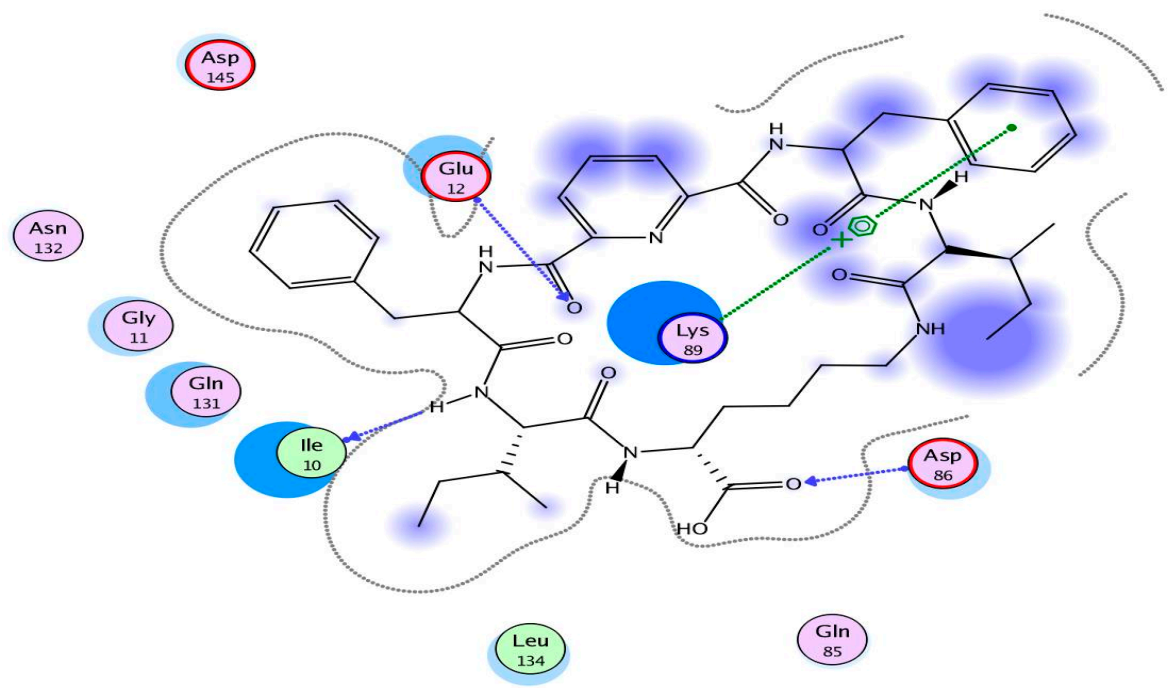

(A)

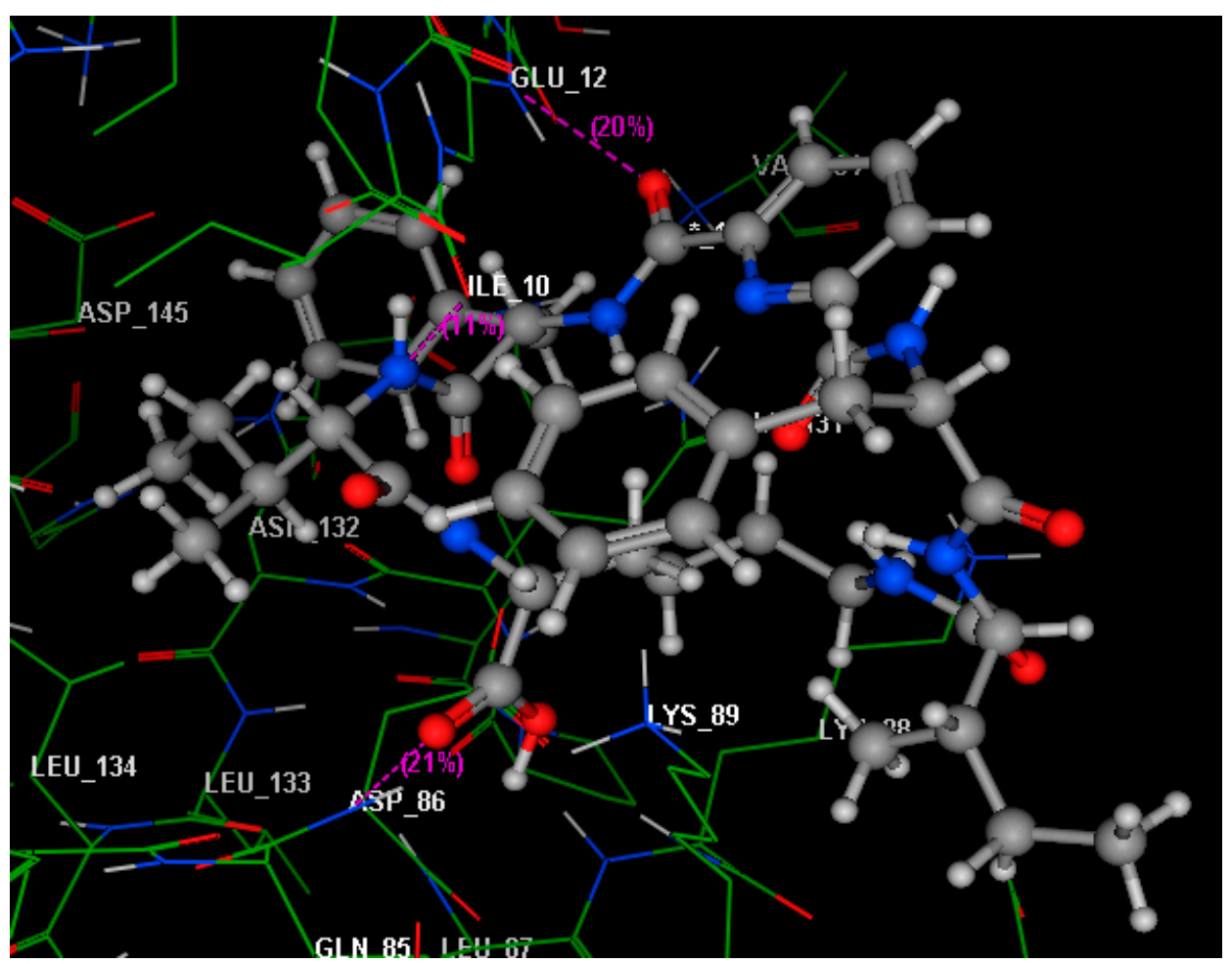

(B)

Figure 6. Docking solution for $5 \mathbf{c}$ in the active site of CDK-2; $(\mathbf{A}, \mathbf{B})$ showing $2 \mathrm{D}$ and 3D ligand-receptor interactions (hydrogen bonds are illustrated as arrows; $\mathrm{C}$ atoms are colored gray, $\mathrm{N}$ blue and $\mathrm{O}$ red).

Finally, it is deduced from molecular docking studies that the good fitting of compound $5 \mathrm{c}$ in the active sites of VEGFR-2 and CDK-2 enzymes via different types of interactions could be attributed to the presence of carboxylic group and cyclic pentapeptide scaffold.

\section{Materials and Methods}

\subsection{Chemistry}

Melting points were determined in an "Electro Thermal" Digital melting point apparatus (Shimadzu, Tokyo, Japan), (model: IA9100). Elemental analysis was found within the acceptable limits of the calculated values (Microanalytical Unit, NRC). Infrared spectra ( $\mathrm{KBr}$ ) were recorded 
on a Nexus 670 FTIR Nicolet, Fourier Transform infrared spectrometer (Perkin Elmer, Hopkinton, MA, USA). Proton nuclear magnetic resonance $\left({ }^{1} \mathrm{H}-\mathrm{NMR}\right)$ spectra were run in $\left[d_{6}\right]$ DMSO on Jeol 270 $\mathrm{MHz}$ or $500 \mathrm{MHz}$ instruments ((Tokyo, Japan). Chemical shifts d are given in ppm. Mass spectra were run on a MAT Finnigan SSQ 7000 spectrometer (Shimadzu, Kyoto, Japan; Model: QP2010 ultra), using the electron impact technique (EI). Analytical thin layer chromatography (TLC) was performed on silica gel aluminum sheets, 60 F254 (E. Merck). Specific optical rotations were measured with a A. Krawss, Optronic, P8000a polarimeter (A. Krüss, Hamburg, Germany), in a 1 dm length observation tube, at the indicated conditions, and according to the equation: $[\mathrm{a}] \mathrm{T} \mathrm{D}=100 . \mathrm{a}=(\mathrm{c} l)$, where $\mathrm{a}=$ observed rotation angle; $\mathrm{D}=$ sodium line $(1=589 \mathrm{~nm}) ; c=$ concentration $(\mathrm{g}=100 \mathrm{~mL})$; $l=$ path length in $\mathrm{dm}$; and $T=$ temperature $\left({ }^{\circ} \mathrm{C}\right)$. The following solvent systems (by volume) were used as eluents for the development of the plates: $S$ : chloroform-methanol-acetic acid (85:10:5); $S_{1}$ : S-petroleum ether (b.p. $\left.40-60{ }^{\circ} \mathrm{C}\right)(1: 1) ; S_{2}$ : S-petroleum ether (b.p. $\left.40-60{ }^{\circ} \mathrm{C}\right)(3: 2)$; $S_{3}$ : S-petroleum ether (b.p. $\left.40-60{ }^{\circ} \mathrm{C}\right)(1: 2)$ and $S_{4}$ : butanol-water-acetic acid-pyridine (120:48:12:40). It is generally known that basic reaction media enhance racemization. However, under the reaction conditions employed in this work, especially short reaction times and temperatures below $\left(0{ }^{\circ} \mathrm{C}\right)$, only negligible racemization was observed.

\subsubsection{Synthesis of $\mathrm{N}^{\alpha}$-dipicolinoyl-bis[dipeptide methyl ester] Derivatives (2a-c)}

Ethyl chloroformate $(0: 2 \mathrm{~mL}, 2 \mathrm{mmol})$ was added to a stirred and cold $\left(-15^{\circ} \mathrm{C}\right)$ dichloromethane solution $(20 \mathrm{~mL})$ of the corresponding $\mathrm{N}^{\alpha}$-dipicolinoyl-bis[amino acid] (1a,b) $(1 \mathrm{mmol})$, containing $N$-methylmorpholine $(0: 2 \mathrm{~mL}, 2 \mathrm{mmol})$. The reaction mixture was stirred for additional $10 \mathrm{~min}$, then a cold dichloromethane solution $(20 \mathrm{~mL})$ of the free amino acid methyl esters, namely, L-isolucine-OMe or L-phenyalanine-OMe $(2 \mathrm{mmol})$, was added. Stirring was maintained for $3 \mathrm{~h}$ at $\left(-15^{\circ} \mathrm{C}\right)$, then for $12 \mathrm{~h}$ at room temperature. The reaction mixture was washed with water, $1 \mathrm{~N}$ sodium bicarbonate, 1 $\mathrm{N}$ potassium hydrogen sulfate and water, and dried over anhydrous calcium chloride. The solvent was evaporated under reduced pressure to dryness, and the obtained oily residue was solidified by trituration with a dry ether- $n$-hexane mixture. The obtained solid was collected by filtration and crystallized from ethanol- $n$-hexane to give the corresponding 2,6-pyridine-bis-dipeptide ester derivatives $(\mathbf{2 a}-\mathbf{c})$, respectively.

$N^{\alpha}$-Dipicolinoyl-bis[L-ILe-L-ILe-methyl ester] (2a). Yield: $80 \%$; m.p. $106-108{ }^{\circ} \mathrm{C},[\alpha]_{\mathrm{D}}^{25}:-71(\mathrm{C}, 0.02$, $\mathrm{MeOH})$. IR (KBr, cm $\left.{ }^{-1}\right): v=3304$ (NH stretching), 3070 ( $\mathrm{CH}$, aromatic), 2966 (CH, aliphatic), 1746 ( $\mathrm{C}=\mathrm{O}$, ester), 1654 and 1440 ( $\mathrm{C}=\mathrm{O}$ amide $\mathrm{I}$ and II, respectively). ${ }^{1} \mathrm{H}-\mathrm{NMR}\left(500 \mathrm{MHz}, \mathrm{ppm}, \mathrm{DMSO}-d_{6}\right)$ : $\delta=8.54-8.51(\mathrm{~m}, 3 \mathrm{H}, \mathrm{Pyr}-\mathrm{H}), 8.40-8.20\left(\mathrm{~s}, 4 \mathrm{H}, 4 \mathrm{NH}, \mathrm{D}_{2} \mathrm{O}\right.$ exchangeable), $4.56(\mathrm{~d}, 2 \mathrm{H}, J=8.8 \mathrm{~Hz}$, $2 \mathrm{NHCH}, \mathrm{L}-\mathrm{Ile}), 4.51(\mathrm{~d}, 2 \mathrm{H}, J=8.8 \mathrm{~Hz}, 2 \mathrm{NHCH}, \mathrm{L}-\mathrm{Ile}), 3.61\left(\mathrm{~s}, 6 \mathrm{H}, 2 \mathrm{OCH}_{3}\right), 3.32-3.30(\mathrm{~m}, 4 \mathrm{H}$,

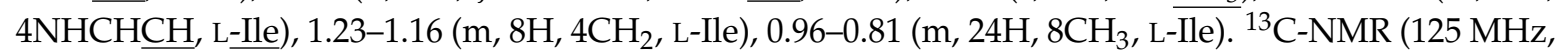
ppm, DMSO- $\left.d_{6}\right): \delta=171.6,170.9\left(2 \mathrm{C}, 2 \mathrm{COOCH}_{3}\right), 162.8(2 \mathrm{C}, 2 \mathrm{CO}), 148.7$ (2CO, Pyridine dicarbonyl), 139.9 (2 C, pyridine, $\mathrm{C}_{2}, \mathrm{C}_{6}$ ), 130.3 (1C, pyridine, $\left.\mathrm{C}_{4}\right), 124.7$ (2 C, pyridine, $\left.\mathrm{C}_{3}, \mathrm{C}_{5}\right), 56.7-54.5$ (4 C, $\mathrm{NHCH}), 50.2\left(2 \mathrm{C}, 2 \mathrm{OCH}_{3}\right), 40.3,39.3$ (4 C, 4NHCHCH, L-Ile), 39.0, $36.0\left(4 \mathrm{C}, 2 \mathrm{CH}_{2}\right), 24.8,11.0$ (8 C, $\underline{8 \mathrm{CH}} \bar{H}_{3}, \mathrm{~L}-\mathrm{Il}$ ) $. \mathrm{MS}(\mathrm{EI}, 70 \mathrm{eV}): \mathrm{m} / z(\%)=648\left(\mathrm{M}^{+}+1,3.62\right), 647\left(\mathrm{M}^{+}, 3.74\right), 591(18.87), 475(100), 330$ (16.79), 302 (14.78), 57 (1.16), 55 (0.53). Analysis for $\mathrm{C}_{33} \mathrm{H}_{53} \mathrm{~N}_{5} \mathrm{O}_{8}$ (647.80): Calcd. C, 61.18; H, 8.25; N, 10.81. Found: C 61.15, H 8.22, N 10.77 .

$N^{\alpha}$-Dipicolinoyl-bis[L-ILe-L-Phe-methyl ester] (2b). Yield: $70 \%$; m.p. $122-124{ }^{\circ} \mathrm{C},[\alpha]_{\mathrm{D}}^{25}:-57(\mathrm{C}, 0.02$, $\mathrm{MeOH})$. IR (KBr, cm $\left.{ }^{-1}\right): v=3330$ (NH stretching), 3087 ( $\mathrm{CH}$, aromatic), 2967 (CH, aliphatic), 1724 ( $\mathrm{C}=\mathrm{O}$, ester), 1653 and 1532 ( $\mathrm{C}=\mathrm{O}$ amide $\mathrm{I}$ and II, respectively). ${ }^{1} \mathrm{H}-\mathrm{NMR}\left(500 \mathrm{MHz}, \mathrm{ppm}, \mathrm{DMSO}-d_{6}\right)$ : $\delta=8.57-8.53$ (m, 3H, Pyr-H), 8.54 (s, 4H, 4NH, D $2 \mathrm{O}$ exchangeable), 8.25-8.20 (m, 10H, Ar-H, L-Phe-ala), $4.56(\mathrm{t}, 2 \mathrm{H}, J=5.02 \mathrm{~Hz}, 2 \mathrm{NHCH}, \mathrm{L}-\mathrm{Phe}-\mathrm{ala}), 4.21$ (d, 2H, J = 8.8 Hz, 2NHCH, $\mathrm{L}-\mathrm{Ile}), 4.16$ (d, $4 \mathrm{H}$, $J=8.1 \mathrm{~Hz}, 2 \mathrm{CH}_{2}$, L-Phe-ala), $3.31\left(\mathrm{~s}, 6 \mathrm{H}, 2 \mathrm{OCH}_{3}\right), 2.98-2.92(\mathrm{~m}, 2 \mathrm{H}, 2 \mathrm{NHCHCH}, \underline{\mathrm{Llle}}), 1.43-1.36(\mathrm{~m}$, $4 \mathrm{H}, 2 \mathrm{CH}_{2}$, L-Ile), 0.96-0.81 (m, 12H, 4CH 3 , L-Ile). ${ }^{13} \mathrm{C}-\mathrm{NMR}\left(125 \mathrm{MHz}, \mathrm{ppm}, \mathrm{DMSO}-d_{6}\right): \delta=172.6$

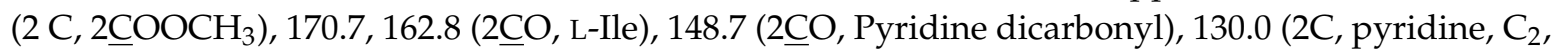


$\left.\mathrm{C}_{6}\right), 128.3$ (1C, pyridine, $\left.\mathrm{C}_{4}\right), 125.7$ (2 C, pyridine, $\left.\mathrm{C}_{3}, \mathrm{C}_{5}\right), 138.2,127.5,126.1,125.4(12 \mathrm{C}, \mathrm{Ph}-\mathrm{C}), 56.8$, $56.3(4 \mathrm{C}, \mathrm{NHCH}), 52.9\left(2 \mathrm{C}, 2 \mathrm{OCH}_{3}\right), 40.4,40.1\left(2 \mathrm{C}, 2 \underline{\mathrm{CH}}_{2}\right.$, L-Phe-ala), 39.8-38.7 (2C, 2NHCHCH, $\left.\mathrm{L}-\mathrm{Nle}\right)$, 37.2-36.1 (2C, $\left.\mathrm{CH}_{2}\right), 24.7,11.0\left(4 \mathrm{C}, 4 \mathrm{CH}_{3}\right.$, L-Ile). MS (EI, $\left.70 \mathrm{eV}\right): m / z(\%)=717\left(\mathrm{M}^{+}+1,0.85\right), 620$ (1.95), 563 (7.83), 461 (100), 404 (3.59), 330 (30.42), 302 (43.45), 69 (8.42), 57 (3.89), 51 (0.96). Analysis for $\mathrm{C}_{39} \mathrm{H}_{49} \mathrm{~N}_{5} \mathrm{O}_{8}$ (715.80): Calcd. C, 65.44; H, 6.90; N, 9.78. Found: C 65.42, H 6.88, N 9.76.

$N^{\alpha}$-Dipicolinoyl-bis[L-Phe-L-ILe- methyl ester] (2c). Yield: 65\%; m.p. $150-152{ }^{\circ} \mathrm{C},[\alpha]_{\mathrm{D}}^{25}:-30(\mathrm{C}, 0.02$, $\mathrm{MeOH})$. IR $\left(\mathrm{KBr}, \mathrm{cm}^{-1}\right): v=3338$ (NH stretching), $3100(\mathrm{CH}$, aromatic), $3000(\mathrm{CH}$, aliphatic), 1780 (C=O, ester), 1695 and 1600 ( $\mathrm{C}=\mathrm{O}$ amide I and II, respectively). ${ }^{1} \mathrm{H}-\mathrm{NMR}$ ( $500 \mathrm{MHz}, \mathrm{ppm}, \mathrm{DMSO}-d_{6}$ ): $\delta=8.90-8.85(\mathrm{~m}, 3 \mathrm{H}, \mathrm{Pyr}-\mathrm{H}), 8.50-8.41\left(\mathrm{~s}, 4 \mathrm{H}, 4 \mathrm{NH}, \mathrm{D}_{2} \mathrm{O}\right.$ exchangeable), 7.60-7.25 (m, 10H, Ar- $\mathrm{H}$, L-Phe-ala), 4.55 (t, 2H, J = 5.02 Hz, 2NHCH, L-Phe-ala), 4.40 (d, 2H, J = $8.8 \mathrm{~Hz}, \mathrm{NHCH}$, L-Ile), 4.25 (d, 4H, $J=8.1 \mathrm{~Hz}, 2 \mathrm{CH}_{2}$, L-Phe-ala), 3.30-3.20 (s, 6H, 2OCH $), 2.90-2.70(\mathrm{~m}, 2 \mathrm{H}, 2 \mathrm{NHCHCH}, \mathrm{L}-\mathrm{Ile}), 1.40-1.25$ (m, $4 \mathrm{H}, 2 \mathrm{CH}_{2}$, L-Ile), $1.05-0.80\left(\mathrm{~m}, 12 \mathrm{H}, 4 \mathrm{CH}_{3}, \mathrm{~L}-\mathrm{Ile}\right) .{ }^{13} \mathrm{C}-\mathrm{NMR}\left(125 \mathrm{MHz}, \mathrm{ppm}, \mathrm{DMSO}-d_{6}\right): \delta=174.0$

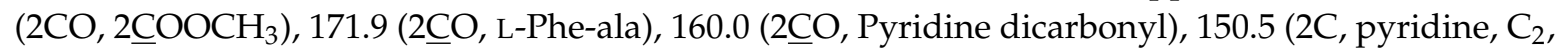
$\left.\mathrm{C}_{6}\right), 138.0$ (1C, pyridine, $\mathrm{C}_{4}$ ), 126.1 (2 C, pyridine, $\mathrm{C}_{3}, \mathrm{C}_{5}$ ), 139.2, 127.7, 126.4, 125.3 (12C, Ph-C), 61.0, $55.0(4 \mathrm{C}, \mathrm{NHCH}), 45.1$ (2C, $2 \underline{\mathrm{CH}}_{2}$, L-Phe-ala), 40.8, 40.5 (2C, 2NHCHCH, L-Ile), 39.0, $37.0\left(2 \mathrm{C}, 2 \mathrm{CH}_{2}\right)$, $24.0\left(2 \mathrm{C}, 2 \mathrm{OCH}_{3}\right), 18.0,11.0\left(4 \mathrm{C}, \mathrm{CH}_{3}, \mathrm{~L}-\mathrm{Ile}\right)$. MS (EI, $\left.70 \mathrm{eV}\right): m / z(\%)=716\left(\mathrm{M}^{+}, 18.00\right), 600(22.12), 484$ (34.00), 350 (29.39), 290 (32.67), 205 (75.81), 125 (100), 91 (82.32), 70 (33), 57 (45.87), 50 (18.85). Analysis for $\mathrm{C}_{39} \mathrm{H}_{49} \mathrm{~N}_{5} \mathrm{O}_{8}$ (715.80): Calcd. C, 65.44; H, 6.90; N, 9.78. Found: C 65.40, H 6.83, N 9.77.

\subsubsection{Synthesis of $\mathrm{N}^{\alpha}$-Dipicolinoyl-bis[dipeptide]derivatives (3a-c)}

To a cold $\left(-15^{\circ} \mathrm{C}\right)$ solution of the corresponding tetrapeptide ester $(\mathbf{2 a}-\mathbf{c})(1 \mathrm{mmol})$ in methanol $(20 \mathrm{~mL})$ with stirring, sodium hydroxide $(1 \mathrm{~N}, 25 \mathrm{~mL})$ was gradually added. The reaction mixture was stirred for $2 \mathrm{~h}$ at the same temperature then for $3 \mathrm{~h}$ at room temperature. The solvent was distilled off under reduced pressure, and the remaining aqueous solution was cooled and acidified with 1 $\mathrm{N}$ hydrochloric acid to $\mathrm{pH}=3$. The obtained solid was filtered off, washed with water, dried and crystallized from ethanol/water to give the corresponding tetrapeptide acids (3a-c), respectively.

$N^{\alpha}$-Dipicolinoyl-bis[L-ILe-L-ILe]acid (3a). Yield: 88\%; m.p. 178-180 ${ }^{\circ} \mathrm{C},[\alpha]_{\mathrm{D}}^{25}:-101(\mathrm{C}, 0.02, \mathrm{MeOH})$. IR $\left(\mathrm{KBr}, \mathrm{cm}^{-1}\right): v=3280$ (NH stretching), $3060(\mathrm{CH}$, aromatic), $2964(\mathrm{CH}$, aliphatic), $1748(\mathrm{C}=\mathrm{O}$, acid), 1651 and 1537 (C=O amide I and II, respectively). ${ }^{1} \mathrm{H}-\mathrm{NMR}\left(500 \mathrm{MHz}, \mathrm{ppm}, \mathrm{DMSO}-d_{6}\right): \delta=12.48$ (s, $2 \mathrm{H}, 2 \mathrm{OH}, \mathrm{D}_{2} \mathrm{O}$ exchangeable), 8.56-8.53 (m, 3H, Pyr-H), 8.39-8.22 (s, 4H, 4NH, $\mathrm{D}_{2} \mathrm{O}$ exchangeable), $4.52(\mathrm{~d}, 4 \mathrm{H}, J=8.8 \mathrm{~Hz}, 4 \mathrm{NHCH}, \mathrm{L}-\mathrm{Ile}), 3.61-3.58(\mathrm{~m}, 4 \mathrm{H}, 4 \mathrm{NHCHCH}, \mathrm{L}-\underline{\mathrm{Ile}}), 1.90-1.15\left(\mathrm{~m}, 8 \mathrm{H}, 4 \mathrm{CH}_{2}\right.$, L-Ile), 0.91-0.82 (m, 24H, 8CH 3 , L-Ile). ${ }^{13} \mathrm{C}-\mathrm{NMR}\left(125 \mathrm{MHz}, \mathrm{ppm}, \mathrm{DMSO}-d_{6}\right): \delta=171.5(2 \mathrm{C}, 2 \mathrm{COOH})$, 162.6 (2CO, L-Ile), 160.5 (2CO, Pyridine dicarbonyl), 139.7 (2C, pyridine, $\mathrm{C}_{2}, \mathrm{C}_{6}$ ), 137.0 (1C, pyridine, $\left.\mathrm{C}_{4}\right), 124.3$ (2 C, pyridine, $\left.\mathrm{C}_{3}, \mathrm{C}_{5}\right), 56.9,51.6(4 \mathrm{C}, 4 \mathrm{NH} \underline{\mathrm{CH}}), 40.3,39.2(4 \mathrm{C}, 4 \mathrm{NHCHC} \mathrm{H}, \mathrm{L}-\mathrm{Ile}), 38.9,36.4$ (4C, $4 \mathrm{CH}_{2}$, L-Ile), 23.8, $10.9\left(8 \mathrm{C}, 8 \mathrm{CH}_{3}\right.$, L-Ile). MS (EI, $\left.70 \mathrm{eV}\right): m / z(\%)=621\left(\mathrm{M}^{+}+1,9.35\right), 537(20.12)$, 509 (100), 330 (25.60), 302 (49.23), 69 (17.34), 59 (52.77), 57 (18.36), $50(0.92)$. Analysis for $\mathrm{C}_{31} \mathrm{H}_{49} \mathrm{~N}_{5} \mathrm{O}_{8}$ (619.70): Calcd. C, 60.08; H, 7.97; N, 11.30. Found: C 60.08, H 7.92, N 11.29.

$N^{\alpha}$-Dipicolinoyl-bis[L-ILe-L-Phe]acid (3b). Yield: 82\%; m.p. 184-186 ${ }^{\circ} \mathrm{C},[\alpha]_{\mathrm{D}}^{25}:-106(\mathrm{C}, 0.02, \mathrm{MeOH}) . \mathrm{IR}$ $\left(\mathrm{KBr}, \mathrm{cm}^{-1}\right): v=3329$ (NH stretching), $3072(\mathrm{CH}$, aromatic), $2965(\mathrm{CH}$, aliphatic), 1726 (C=O, acid), 1652 and 1532 (C=O amide I and II, respectively). ${ }^{1} \mathrm{H}-\mathrm{NMR}\left(500 \mathrm{MHz}, \mathrm{ppm}, \mathrm{DMSO}-d_{6}\right): \delta=12.65$ (s, $2 \mathrm{H}, 2 \mathrm{OH}, \mathrm{D}_{2} \mathrm{O}$ exchangeable), 8.52-8.43 (m, 3H, Pyr- $\left.\mathrm{H}\right), 8.40-8.20\left(\mathrm{~s}, 4 \mathrm{H}, 4 \mathrm{NH}, \mathrm{D}_{2} \mathrm{O}\right.$ exchangeable), 7.23-7.08 (m, 10H, Ar-H, L-Phe-ala), 4.45 (t, 2H, $J=5.02 \mathrm{~Hz}, 2 \mathrm{NHCH}$, L-Phe-ala), 4.40 (d, 2H, $J=8.8 \mathrm{~Hz}$, 2NHCH, L-Ile), 4.31 (d, 4H, J = 8.1 Hz, 2CH , L-Phe-ala), 3.00-2.91 (m, 2H, 2NHCHCH, L-Ile), 1.9-1.5

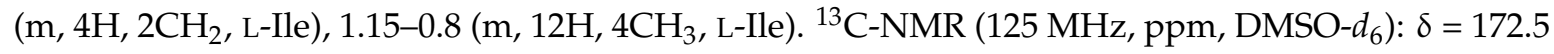
(2C, 2COOH), 169.1 (2C, 2CO , L-Ile), 162.6 (2C, 2으, Pyridine dicarbonyl), 148.5 (2C, pyridine, $\mathrm{C}_{2}, \mathrm{C}_{6}$ ), 139.7 (1C, pyridine, $\left.\mathrm{C}_{4}\right), 126.00$ (2 C, pyridine, $\mathrm{C}_{3}, \mathrm{C}_{5}$ ), 139.0, 127.2, 126.4, 125.6 (12C, Ph-C), 56.9, 53.2

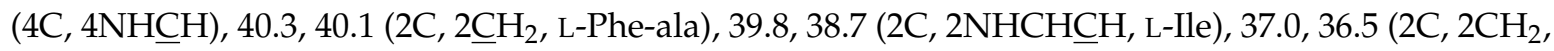
L-Ile), 23.8, $10.9\left(4 \mathrm{C}, 4 \underline{\mathrm{CH}}_{3}\right.$, L-Ile). MS (EI, $\left.70 \mathrm{eV}\right): m / z(\%)=689\left(\mathrm{M}^{+}+1,1.78\right), 688\left(\mathrm{M}^{+}, 4.41\right), 687$ 
(8.50), 625 (71.56), 524 (100), 495 (92.50), 477 (51.54), 330 (56.17), 86 (36.66), 57 (12.05), 50 (2.34). Analysis for $\mathrm{C}_{37} \mathrm{H}_{45} \mathrm{~N}_{5} \mathrm{O}_{8}$ (687.80): C, 64.61; H, 6.59; N, 10.18. Found: C 64.58, H 6.57, N 10.17.

$N^{\alpha}$-Dipicolinoyl-bis[L-Phe-L-ILe]acid (3c). Yield: 77\%; m.p. 202-204 ${ }^{\circ} \mathrm{C},[\alpha]_{\mathrm{D}}^{25}:-70(\mathrm{C}, 0.02, \mathrm{MeOH}) . \mathrm{IR}$ $\left(\mathrm{KBr}, \mathrm{cm}^{-1}\right): v=3321$ (NH stretching), $3031(\mathrm{CH}$, aromatic), $2967(\mathrm{CH}$, aliphatic), $1725(\mathrm{C}=\mathrm{O}$, acid), 1655 and 1530 (C=O amide I and II, respectively). ${ }^{1} \mathrm{H}-\mathrm{NMR}\left(500 \mathrm{MHz}, \mathrm{ppm}, \mathrm{DMSO}-d_{6}\right): \delta=12.70$ (s, $2 \mathrm{H}, 2 \mathrm{OH}, \mathrm{D}_{2} \mathrm{O}$ exchangeable), 9.60-8.50 (m, 3H, Pyr-H), 8.23-8.09 (s, 4H, 4NH, $\mathrm{D}_{2} \mathrm{O}$ exchangeable), 7.44-7.12 (m, 10H, Ar-H, L-Phe-ala), 4.90 (t, $J=5.02 \mathrm{~Hz}, 2 \mathrm{H}, 2 \mathrm{NHCH}$, L-Phe-ala), 4.29 (d, 2H, J = $8.8 \mathrm{~Hz}$, 2NHCH, L-Ile), 4.00 (d, 4H, J = 8.1 Hz, 2CH , L-Phe-ala), 3.34-3.13 (m, 2H, 2NHCHCH, L-Ile), 1.23-1.16 (m, 4H, 2CH $\mathrm{CH}_{2}$ L-Ile), $0.92-0.80\left(\mathrm{~m}, 12 \mathrm{H}, 4 \mathrm{CH}_{3}\right.$, L-Ile). ${ }^{13} \mathrm{C}-\mathrm{NMR}\left(125 \mathrm{MHz}, \mathrm{ppm}, \mathrm{DMSO}-d_{6}\right): \delta=173.2$ (2C, 2COOH), 172.6, 170.8 (2CO, L-Phe-ala), 164.9, 163.4 (2CO, Pyridine dicarbonyl), 148.6 (2C, pyridine, $\mathrm{C}_{2}, \mathrm{C}_{6}$ ), 139.6 (1C, pyridine, $\mathrm{C}_{4}$ ), 125.6 (2 C, pyridine, $\mathrm{C}_{3}, \mathrm{C}_{5}$ ), 138.15 126.8, 126.3, 125.8 (12C, Ph-C), 59.8, 56.4 (4C, $4 \mathrm{NH} \underline{\mathrm{CH}}), 43.1,40.4$ (2C, $2 \underline{\mathrm{CH}}_{2}$, L-Phe-ala), 39.8, 39.2 (2C, 2NHCHCH, L-Ile), 39.0, 36.0 (2C, $\left.\underline{\mathrm{CH}}_{2}\right), 24.7,11.0\left(4 \mathrm{C}, \underline{\mathrm{CH}_{3}}, \mathrm{~L}-\mathrm{Ile}\right)$. MS (EI, $\left.70 \mathrm{eV}\right): \mathrm{m} / z(\%)=689\left(\mathrm{M}^{+}+1,2.05\right), 688\left(\mathrm{M}^{+}, 4.22\right), 669$ (8.33), 625 (22.60), 558 (67.30), 529 (49.57), 461 (18.79), 416 (26.29), 370 (100), 302 (11.74), 86 (29.02), 57 (15.14), 51 (5.33). Analysis for $\mathrm{C}_{37} \mathrm{H}_{45} \mathrm{~N}_{5} \mathrm{O}_{8}$ (687.80): Calcd. C, 64.61; $\mathrm{H}, 6.59 ; \mathrm{N}, 10.18$. Found: $\mathrm{C}$ 64.60, H 6.60, N 10.18 .

3.1.3. Synthesis of Cyclo-( $\mathrm{N}^{\alpha}$-Dipicolinoyl)-bis-[dipeptide]-L-Lys-OMe (cyclic pentapeptide methyl esters) (4a-c)

To a stirred and cold $\left(-15{ }^{\circ} \mathrm{C}\right)$ dichloromethane solution $(20 \mathrm{~mL})$ of the corresponding $\mathrm{N}^{\alpha}$-dipicolinoyl-bis[dipeptide] (3a-c) $(1 \mathrm{mmol})$, containing $N$-methylmorpholine $(0: 2 \mathrm{~mL}, 2 \mathrm{mmol})$, ethyl chloroformate $(0.2 \mathrm{~mL}, 2 \mathrm{mmol})$ was added and stirred for $20 \mathrm{~min}$. To the reaction mixture, free L-lysine methyl ester $(1 \mathrm{mmol})$ in dichloromethane $(20 \mathrm{~mL})$ was added at $\left(-15^{\circ} \mathrm{C}\right)$ with stirring. Stirring was maintained for $3 \mathrm{~h}$, at $\left(-15^{\circ} \mathrm{C}\right)$, then for $12 \mathrm{~h}$ at room temperature. The reaction mixture was washed with water, $1 \mathrm{~N}$ sodium bicarbonate, $1 \mathrm{~N}$ potassium hydrogen sulfate and water, and then dried over anhydrous calcium chloride. The solvent was evaporated under reduced pressure to dryness, and the obtained oily residue was solidified by trituration with dry ether- $n$-hexane mixture. The crude product was purified by preparative thin layer chromatography using S3 as eluent to give the corresponding cyclic pentapeptide methyl esters $(\mathbf{4 a}-\mathbf{c})$, respectively.

Cyclo-(N ${ }^{\alpha}$-dipicolinoyl)-bis-[L-ILe-L-ILe]-L-Lys-OMe (4a). Yield: 78\%; m.p. 77-79 ${ }^{\circ} \mathrm{C},[\alpha]_{\mathrm{D}}^{25}:-13(\mathrm{C}, 0.02$, $\mathrm{MeOH})$. IR (KBr, cm $\left.{ }^{-1}\right): v=3398$ (NH stretching), 2945 ( $\mathrm{CH}$, aromatic), 2867, 2863 (CH, aliphatic), 1642 (C=O, ester), 1530, 1454 and 1396 (C=O amide I, II and III, respectively). ${ }^{1} \mathrm{H}-\mathrm{NMR}$ (500 MHz, ppm, DMSO- $\left.d_{6}\right): \delta=8.90-8.76(\mathrm{~m}, 3 \mathrm{H}, \mathrm{Pyr}-\mathrm{H}), 8.32,8.21\left(\mathrm{~s}, 6 \mathrm{H}, 6 \mathrm{NH}, \mathrm{D}_{2} \mathrm{O}\right.$ exchangeable), $4.66(\mathrm{~d}, 4 \mathrm{H}$, $J=8.8 \mathrm{~Hz}, 4 \mathrm{NHCH}, \mathrm{L}-\mathrm{Ile}), 4.45(\mathrm{t}, 1 \mathrm{H}, J=9.7 \mathrm{~Hz}, \mathrm{CH}, \mathrm{L}-\mathrm{Lys}), 3.84\left(\mathrm{~s}, 3 \mathrm{H}, \mathrm{OCH}_{3}\right), 3.57-3.55\left(\mathrm{~m}, 2 \mathrm{H}, \mathrm{CH}_{2}\right.$, $\left.\mathrm{NHCH}_{2}\right), 2.70-2.55(\mathrm{~m}, 4 \mathrm{H}, 4 \mathrm{NHCHCH}, \underline{\mathrm{L}}-\mathrm{Ile}), 2.00-1.45\left(\mathrm{~m}, 6 \mathrm{H}, 3 \mathrm{CH}_{2}, \mathrm{NHCH}_{2} \mathrm{CH}_{2} \mathrm{CH}_{2} \mathrm{CH}_{2} \mathrm{CHNH}\right)$, $1.30-1.15\left(\mathrm{~m}, 8 \mathrm{H}, 4 \mathrm{CH}_{2}\right.$, L-Ile), $1.05-0.80\left(\mathrm{~m}, 24 \mathrm{H}, 8 \mathrm{CH}_{3}\right)$. MS (EI, $\left.70 \mathrm{eV}\right): m / z(\%)=743\left(\mathrm{M}^{+}, 13.34\right), 568$ (10.65), 460 (11.38), 129 (23.58), 96 (28.34), 85 (34.33), 69 (69.08), 60 (74.04), 57 (77.97), 55 (100), 50 (14.79). Analysis for $\mathrm{C}_{38} \mathrm{H}_{61} \mathrm{~N}_{7} \mathrm{O}_{8}$ (743.90): Calcd. C, 61.35; $\mathrm{H}, 8.26 ; \mathrm{N}, 13.18$. Found: $\mathrm{C} 61.30, \mathrm{H}$ 8.22, N 13.14.

Cyclo-(N ${ }^{\alpha}$-Dipicolinoyl)-bis-[L-ILe-L-Phe]-L-Lys-OMe (4b). Yield: $81 \%$; m.p. $104-106^{\circ} \mathrm{C},[\alpha]_{\mathrm{D}}^{25}:-28(\mathrm{C}$, 0.02, $\mathrm{MeOH})$. IR (KBr, cm $\left.{ }^{-1}\right): v=3368$ (NH stretching), $2945(\mathrm{CH}$, aromatic), $2835(\mathrm{CH}$, aliphatic), 1657 (C=O, ester), 1528, 1425 and 1222 (C=O amide I, II and III, respectively). ${ }^{1} \mathrm{H}-\mathrm{NMR}$ (500 MHz, ppm, DMSO- $\left.d_{6}\right): \delta=8.60-8.58(\mathrm{~m}, 3 \mathrm{H}, \mathrm{Pyr}-\mathrm{H}), 8.43,8.21\left(2 \mathrm{~s}, 6 \mathrm{H}, 6 \mathrm{NH}, \mathrm{D}_{2} \mathrm{O}\right.$, exchangeable), 7.22-7.10 $(\mathrm{m}, 10 \mathrm{H}, \mathrm{Ar}-\mathrm{H}, \mathrm{L}-\mathrm{Phe}-\mathrm{ala}), 4.46(\mathrm{t}, 2 \mathrm{H}, J=5.02 \mathrm{~Hz}, 2 \mathrm{NHCH}, \mathrm{L}-\mathrm{Phe}-\mathrm{ala}), 4.44(\mathrm{~d}, 2 \mathrm{H}, J=8.8 \mathrm{~Hz}$, 2NHCH, L-Ile), $4.43(\mathrm{t}, 1 \mathrm{H}, \mathrm{CH}, \mathrm{L}-\mathrm{Lys}), 4.41\left(\mathrm{~d}, 4 \mathrm{H}, J=8.1 \mathrm{~Hz}, 2 \mathrm{CH}_{2}\right.$, L-Phe-ala), 3.61-3.57 (m, 2H, $\mathrm{CH}_{2}, \mathrm{NHCH}_{2}$, L-Lys), 3.32 (s, 3H, OCH 3 ), 3,00-2.95 (m, 2H, 2NHCHCH, L-Ile), 1.9-1.5 (m, 6H, 3CH $\left.\mathrm{NHCH}_{2} \mathrm{CH}_{2} \mathrm{CH}_{2} \mathrm{CH}_{2} \mathrm{CHNH}\right), 1.4-1.35$ (m, $4 \mathrm{H}, 2 \mathrm{CH}_{2}$, L-Ile), 0.91-0.81 (m, 12H, 4CH, L-Ile). ${ }^{13} \mathrm{C}-\mathrm{NMR}$ $\left(125 \mathrm{MHz}, \mathrm{ppm}, \mathrm{DMSO}-d_{6}\right): \delta=171.6\left(\mathrm{COOCH}_{3}\right), 170.6,162.7(6 \mathrm{C}, 6 \underline{\mathrm{CO}}), 148.5\left(2 \mathrm{C}\right.$, pyridine, $\left.\mathrm{C}_{2}, \mathrm{C}_{6}\right)$, 139.8 (1C, pyridine, $\mathrm{C}_{4}$ ), 127.1 (2 C, pyridine, $\mathrm{C}_{3}, \mathrm{C}_{5}$ ), 138.8, 127.6, 126.8, 125.2 (12C, Ph-C), 56.9-51.7

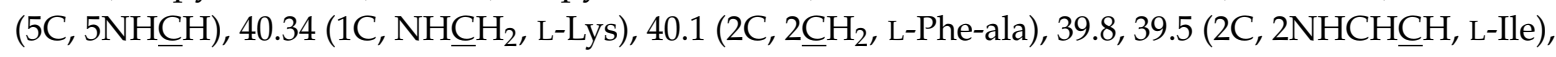


39.22, $36.39\left(5 \mathrm{C}, 5 \mathrm{CH}_{2}\right), 23.2\left(1 \mathrm{C}, \mathrm{OCH}_{3}\right), 15.3,11.0\left(4 \mathrm{C}, 4 \underline{C H}_{3}, \mathrm{~L}-\mathrm{Ile}\right) . \mathrm{MS}(\mathrm{EI}, 70 \mathrm{eV}): \mathrm{m} / z(\%)=796$ $\left(\mathrm{M}^{+}, 1.05\right), 715$ (9.49), 659 (5.53), 509 (71.40), 449 (9.59), 347 (26.17), 302 (100), 180 (62.98), 86 (48.45), 69 (30.62), 52 (0.14). Analysis for $\mathrm{C}_{44} \mathrm{H}_{57} \mathrm{~N}_{7} \mathrm{O}_{8}$ (811.96): Calcd. C, 65.09; H, 7.08; N, 12.08. Found: C, 65.00; $\mathrm{H}, 7.00 ; \mathrm{N}, 12.00$.

Cyclo-(N ${ }^{\alpha}$-Dipicolinoyl)-bis-[L-Phe-L-ILe]-L-Lys-OMe (4c). Yield: 75\%; m.p. $140-142{ }^{\circ} \mathrm{C},[\alpha]_{\mathrm{D}}^{25}:-55(\mathrm{C}$, 0.02, MeOH). IR ( $\left.\mathrm{KBr}, \mathrm{cm}^{-1}\right): v=3320$ (NH stretching), $3065(\mathrm{CH}$, aromatic), 2965 (CH, aliphatic), 1783 (C=O, ester), 1663, 1527 and 1447 (C=O amide I, II and III, respectively). ${ }^{1} \mathrm{H}-\mathrm{NMR}$ (500 MHz, ppm, DMSO- $\left.d_{6}\right): \delta=8.80-8.78(\mathrm{~m}, 3 \mathrm{H}, \mathrm{Pyr}-\mathrm{H}), 8.63,8.51\left(\mathrm{~s}, 6 \mathrm{H}, 6 \mathrm{NH}, \mathrm{D}_{2} \mathrm{O}\right.$ exchangeable), 7.52-7.10 (m, $10 \mathrm{H}, \mathrm{Ar}-\mathrm{H}, \mathrm{L}-\mathrm{Phe}-\mathrm{ala}), 4.65$ (t, 2H, $J=5.02 \mathrm{~Hz}, 2 \mathrm{NHCH}, \mathrm{L}-\mathrm{Phe}-\mathrm{ala}), 4.50$ (d, 2H, $J=8.8 \mathrm{~Hz}, 2 \mathrm{NHCH}$, L-Ile), 4.40 (t, 1H, J = 9.7 Hz, CH, L-Lys), 4.36 (d, 4H, J = 8.1 Hz, 2CH2, L-Phe-ala), 3.82-3.74 (m, $2 \mathrm{H}, \mathrm{CH}_{2}, \mathrm{NHCH}_{2}$, L-Lys), 3.52 (s, 3H, $\mathrm{OCH}_{3}$ ), 3,20-3.05 (m, 2H, 2NHCHCH, L-Ile), 1.85-1.45 (m, $\left.6 \mathrm{H}, 3 \mathrm{CH}_{2}, \mathrm{NHCH}_{2} \mathrm{CH}_{2} \mathrm{CH}_{2} \mathrm{CH}_{2} \mathrm{CHNH}\right), \overline{1.38}-1.36\left(\mathrm{~m}, 4 \mathrm{H}, 2 \mathrm{CH}_{2}, \mathrm{~L}-\mathrm{Ile}\right), 0.90-0.75\left(\mathrm{~m}, 12 \mathrm{H}, 4 \mathrm{CH}_{3}\right.$, L-Ile). ${ }^{13} \mathrm{C}-\mathrm{NMR}\left(125 \mathrm{MHz}, \mathrm{ppm}, \mathrm{DMSO}-d_{6}\right): \delta=174.2\left(\mathrm{COOCH}_{3}\right), 172.8,168.0(6 \mathrm{C}, 6 \mathrm{CO}), 150.6$ (2C, pyridine, $\left.\mathrm{C}_{2}, \mathrm{C}_{6}\right), 140.2$ (1C, pyridine, $\left.\mathrm{C}_{4}\right), 125.6$ (2 C, pyridine, $\left.\mathrm{C}_{3}, \mathrm{C}_{5}\right), 138.2$ 127.0, 126.2, $125.4(12 \mathrm{C}$, Ph-C), 60.3-55.9 (5C, 5NHChH), 48.40 (1C, $\mathrm{NHCH}_{2}$, L-Lys), 45.0 (2C, 2대 ${ }_{2}$, L-Phe-ala), 42.0, 41.5 (2C,

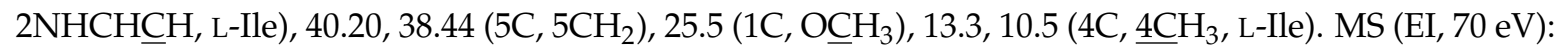
$m / z(\%)=813\left(\mathrm{M}^{+}+1,25.06\right), 812\left(\mathrm{M}^{+}, 42.13\right), 755$ (33.32), $720(37.79), 698(22.27), 596(21.32), 571$ (30.36), 543 (32.52), 495 (20.68), 458 (25.09), 430 (56.12), 370 (100), 91 (14.24), 69 (10.40), 57 (3.84), 50 (3.80). Analysis for $\mathrm{C}_{44} \mathrm{H}_{57} \mathrm{~N}_{7} \mathrm{O}_{8}$ (811.96). Calcd. C, 65.09; H, 7.08; N, 12.08. Found: C, 65.00; H, 7.00; N, 12.00 .

\subsubsection{Synthesis of Cyclo-( $\mathrm{N}^{\alpha}$-dipicolinoyl)-bis[dipeptide]-L-Lys-OH (cyclic pentapeptides) (5a-c)}

To a stirred and cold methanolic solution $\left(-5^{\circ} \mathrm{C}, 20 \mathrm{~mL}\right)$ of the corresponding cyclic pentapeptide methyl ester $(4 \mathbf{a}-\mathbf{c})(1 \mathrm{mmol})$, sodium hydroxide $(1 \mathrm{~N}, 25 \mathrm{~mL})$ was gradually added. The reaction mixture was stirred for $2 \mathrm{~h}$ at the same temperature, then for $3 \mathrm{~h}$ at room temperature. The solvent was distilled off under reduced pressure, and the remaining aqueous solution was cooled and acidified with $1 \mathrm{~N}$ hydrochloric acid to $\mathrm{pH}=3$. The obtained solid was filtered off, washed with water, dried and crystallized from ethanol-water to give the corresponding cyclic pentapeptides (5a-c), respectively.

Cyclo-(N ${ }^{\alpha}$-dipicolinoyl)-bis [L-ILe-L-ILe]-L-Lys-OH (5a). Yield: 69\%; m.p. 108-110 ${ }^{\circ} \mathrm{C},[\alpha]_{\mathrm{D}}^{25}:-21(\mathrm{C}$, 0.02, $\mathrm{MeOH})$. IR ( $\left.\mathrm{KBr}, \mathrm{cm}^{-1}\right): v=3317$ (NH stretching), 3081 ( $\mathrm{CH}$, aromatic), 2967 (CH, aliphatic), 1724 (C=O, acid), 1655, 1533 and 1456 (C=O amide I, II and III, respectively). ${ }^{1} \mathrm{H}-\mathrm{NMR}(500 \mathrm{MHz}$, ppm, DMSO- $\left.d_{6}\right): \delta=12.60\left(\mathrm{~s}, 1 \mathrm{H}, \mathrm{OH}, \mathrm{D}_{2} \mathrm{O}\right.$ exchangeable), 8.51-8.49 (m, 3H, Pyr-H), 8.46, 8.20 (2s, $6 \mathrm{H}, 6 \mathrm{NH}, \mathrm{D}_{2} \mathrm{O}$ exchangeable), 4.70- (d, $\left.4 \mathrm{H}, J=8.8 \mathrm{~Hz}, 4 \mathrm{NHCH}, \mathrm{L}-\mathrm{Ile}\right), 4.18(\mathrm{t}, 1 \mathrm{H}, J=9.7 \mathrm{~Hz}, \mathrm{CH}$, L-Lys), 3.31-3.28 (m, 2H, $\left.\mathrm{CH}_{2}, \mathrm{NHCH}_{2}, \mathrm{~L}-\mathrm{Lys}\right), 1.94-1.92$ (m, 4H, 4NHCHCH$), 1.90-1.88$ (m, 6H, 3CH $\left.\mathrm{NHCH}_{2} \mathrm{CH}_{2} \mathrm{CH}_{2} \mathrm{CH}_{2}\right), 1.50-1.16\left(\overline{\mathrm{m}, 8 \mathrm{H}}, 4 \mathrm{CH}_{2}\right.$, L-Ile), 0.96-0.81 (m, 24H, 8CH 3 , L-Ile). MS (EI, $\left.70 \mathrm{eV}\right)$ : $m / z(\%)=730\left(\mathrm{M}^{+}, 22.28\right), 682$ (35.08), 621 (48.97), 565 (35.49), 395 (44.98), 285 (31.09), 244 (25.03), 69 (68.36), 57 (100), 51 (14.03). Analysis for $\mathrm{C}_{37} \mathrm{H}_{59} \mathrm{~N}_{7} \mathrm{O}_{8}$ (729.90): Calcd. C, 60.88; H, 8.15; N, 13.43. Found: C 60.84, H 8.11, N 13.40 .

Cyclo-(N ${ }^{\alpha}$-dipicolinoyl)-bis [L-ILe-L-Phe]-L-Lys-OH (5b). Yield: 73\%; m.p. $128-130{ }^{\circ} \mathrm{C},[\alpha]_{\mathrm{D}}^{25}:-35(\mathrm{C}$, 0.02, $\mathrm{MeOH})$. IR (KBr, cm $\left.{ }^{-1}\right): v=3312$ (NH stretching), 3067 (CH, aromatic), 2965 (CH, aliphatic), 1725 (C=O, acid), 1655, 1531 and 1448 (C=O amide I, II and III, respectively). ${ }^{1} \mathrm{H}-\mathrm{NMR}(500 \mathrm{MHz}$, ppm, DMSO- $\left.d_{6}\right): \delta=12.70\left(\mathrm{~s}, 1 \mathrm{H}, \mathrm{OH}, \mathrm{D}_{2} \mathrm{O}\right.$ exchangeable), 8.43-8.38 (3H, Pyr-H), 8.22, 8.20 (2s, 6H, $6 \mathrm{NH}, \mathrm{D}_{2} \mathrm{O}$ exchangeable), 7.23-7.08 (m, 10H, Ar-H, L-Phe-ala), 4.45-4.42 (m, 5H, 5CH ), 4.40 (t, $4 \mathrm{H}, J$

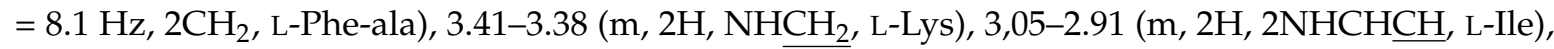
1.95-1.50 (m, 6H, 3CH, $\left.\mathrm{NHCH}_{2} \mathrm{CH}_{2} \mathrm{CH}_{2} \mathrm{CH}_{2} \mathrm{CHNH}\right), 1.18$ (m, 4H, 2CH2, L-Ile), 0.90-0.80 (m, 12H, $\left.4 \mathrm{CH}_{3}, \mathrm{~L}-\mathrm{Ile}\right) .{ }^{13} \mathrm{C}-\mathrm{NMR}\left(125 \mathrm{MHz}, \mathrm{ppm}, \mathrm{DMSO}-d_{6}\right): \delta=172.6$ (OOH), 170.4, $170.3(4 \mathrm{C}, 4 \underline{\mathrm{CO}}), 162.7$ (2CO , Pyridine dicarbonyl), 148.6 (2C, pyridine, $\mathrm{C}_{2}, \mathrm{C}_{6}$ ), 139.7 (1C, pyridine, $\mathrm{C}_{4}$ ), 128.1 (2 C, pyridine, $\left.\mathrm{C}_{3}, \mathrm{C}_{5}\right), 139.0,127.8,126.7,125.5$ (12C, Ph-C), 57.0, 53.3 (5C, 5 NHCH), 40.4 (1C, NHC $H_{2}$, L-Lys), 40.1, 39.8 (2C, 2 $\mathrm{CH}_{2}$, L-Phe-ala), 39.5, 39.3 (2C, 2NHCHCH, L-Ile), 39.0, $36.5\left(5 \mathrm{C}, 5 \mathrm{CH}_{2}\right), 23.9,11.0\left(4 \mathrm{C}, \underline{4 \mathrm{CH}_{3}}\right.$, 
L-Ile). MS (EI, $70 \mathrm{eV}): m / z(\%)=797\left(\mathrm{M}^{+}, 16.93\right), 708$ (16.34), 621 (28.74), 562 (22.24), 504 (24.21), 321 (18.01), 281 (17.72), 170 (18.21), 85 (19.59), 71 (40.85), 61 (20.37), 59 (52.07), 57 (100), 51 (17.22). Analysis for $\mathrm{C}_{43} \mathrm{H}_{55} \mathrm{~N}_{7} \mathrm{O}_{8}$ (797.90): Calcd. C, 64.72; H, 6.95; N, 12.29. Found: C 64.69, H 6.94, N 12.27.

Cyclo-(N ${ }^{\alpha}$-dipicolinoyl)-bis [L-Phe-L-ILe]-L-Lys-OH (5c). Yield: 81\%; m.p. 178-180 ${ }^{\circ} \mathrm{C},[\alpha]_{\mathrm{D}}^{25}:-30(\mathrm{C}, 0.02$, $\mathrm{MeOH})$. IR $\left(\mathrm{KBr}, \mathrm{cm}^{-1}\right): v=3327$ (NH stretching), $3065(\mathrm{CH}$, aromatic), $2965(\mathrm{CH}$, aliphatic), 1721 (C=O, acid), 1659, 1527 and 1448 ( $\mathrm{C}=\mathrm{O}$ amide I, II and III, respectively). ${ }^{1} \mathrm{H}-\mathrm{NMR}$ (500 MHz, ppm, DMSO- $\left.d_{6}\right): \delta=12.85\left(\mathrm{~s}, 1 \mathrm{H}, \mathrm{OH}, \mathrm{D}_{2} \mathrm{O}\right.$ exchangeable), 8.60-8.45 (m, 3H, Pyr-H), 8.33, $8.25(2 \mathrm{~s}, 6 \mathrm{H}$, $6 \mathrm{NH}, \mathrm{D}_{2} \mathrm{O}$ exchangeable), 7.55-7.10 (m, 10H, Ar-H, L-Phe-ala), 4.60-4.45 (m, 5H, 5다), $4.35(\mathrm{t}, 4 \mathrm{H}$, $J=8.1 \mathrm{~Hz}, 2 \mathrm{CH}_{2}$, L-Phe-ala), 3.50-3.45 (m, 2H, $1 \mathrm{CH}_{2}, \mathrm{NHCH}_{2}$, L-Lys), 3,25-3.10 (m, 2H, 2NHCHCH, L-Ile), 2.00-1.70 (m, 6H, 3CH, $\left.\mathrm{NHCH}_{2} \mathrm{CH}_{2} \mathrm{CH}_{2} \mathrm{CH}_{2}\right), 1.30\left(\mathrm{~m}, 4 \mathrm{H}, 2 \mathrm{CH}_{2}\right.$, L-Ile), 1.10-0.70 (m, $12 \mathrm{H}$, $\left.4 \mathrm{CH}_{3}, \mathrm{~L}-\mathrm{Ile}\right) .{ }^{13} \mathrm{C}-\mathrm{NMR}\left(125 \mathrm{MHz}, \mathrm{ppm}, \overline{\left.\mathrm{DMSO}-d_{6}\right): \delta}=176.0(\underline{\mathrm{COOH}}), 174.5,172.8\right.$ (4드, L-Phe-ala, L-Ile), 169.0 (2COC, Pyridine dicarbonyl), 150.3 (2C, pyridine, $\left.\mathrm{C}_{2}, \mathrm{C}_{6}\right), 140.9$ (1C, pyridine, $\left.\mathrm{C}_{4}\right), 125.9$ (2C, pyridine, $\left.\mathrm{C}_{3}, \mathrm{C}_{5}\right), 138.4,127.0,126.5,125.7$ (12C, Ph-C), 61.0, 56.3 (5C, 5NHCH$), 45.5$ (1C, $\left.\mathrm{NH}_{2} \mathrm{H}_{2}\right), 46.1$, 40.8 (2C, $2 \underline{\mathrm{CH}}_{2}$, L-Phe-ala), 39.5, 39.3 (2C, $\left.2 \mathrm{NHCH} \underline{\mathrm{CH}}\right), 38.0,36.5\left(5 \mathrm{C}, 5 \mathrm{CH}_{2}\right), 24.5,11.0\left(4 \mathrm{C}, \underline{4} \mathrm{CH}_{3}\right.$, L-Ile). MS (EI, $70 \mathrm{eV}): m / z(\%)=797\left(\mathrm{M}^{+}, 6.08\right), 681$ (6.44), 636 (37.92), 621 (100), 588 (8.55), 565 (44.24), 304 (7.27), 89 (7.27), 67 (4.61), 57 (11.72), 55 (11.41). Analysis for $\mathrm{C}_{43} \mathrm{H}_{55} \mathrm{~N}_{7} \mathrm{O}_{8}$ (797.90): Calcd. C, 64.72; H, 6.95; N, 12.29. Found: C 64.70, H 6.6.90, N 12.22.

3.1.5. Synthesis of Cyclo-( $\mathrm{N}^{\alpha}$-dipicolinoyl)-bis[dipeptide]-L-Lys- $\mathrm{NHNH}_{2}$ (cyclic pentapeptide hydrazides) (6a-c)

A mixture of cyclic pentapeptide methyl esters $(4 \mathbf{a}-\mathbf{c})(1 \mathrm{mmol})$ and anhydrous hydrazine hydrate $(0.35 \mathrm{~mL}, 10 \mathrm{mmol})$ in methanol $(20 \mathrm{~mL})$ was stirred at room temperature for $30 \mathrm{~min}$., and then it was for $3 \mathrm{~h}$. The reaction mixture was evaporated under reduced pressure; the obtained residue oil was solidified with ether, filtered off and crystallized from methanol/ether to afford the corresponding cyclic pentapeptide hydrazides $(\mathbf{6 a}-\mathbf{c})$, respectively.

Cyclo-(N ${ }^{\alpha}$-dipicolinoyl)-bis[L-ILe-L-ILe]-L-Lys-NHNH 2 (6a). Yield: $60 \%$; m.p. $180-182{ }^{\circ} \mathrm{C},[\alpha]_{\mathrm{D}}^{25}:-12(\mathrm{C}$, 0.02, MeOH). IR $\left(\mathrm{KBr}, \mathrm{cm}^{-1}\right): v=3280(\mathrm{NH}$ stretching), 30,867 (CH, aromatic), $2964(\mathrm{CH}$, aliphatic), 1644, 1532, 1450 and 1383 (C=O amide I, II, III and IV, respectively). ${ }^{1} \mathrm{H}-\mathrm{NMR}$ (500 MHz, ppm, DMSO- $\left.d_{6}\right): \delta=9.02\left(\mathrm{~s}, 1 \mathrm{H}, \mathrm{CONH}, \mathrm{D}_{2} \mathrm{O}\right.$ exchangeable), $8.75-8.70(3 \mathrm{H}, \mathrm{Pyr}-\mathrm{H}), 8.20,8.10(2 \mathrm{~s}, 6 \mathrm{H}, 6 \mathrm{NH}$, $\mathrm{D}_{2} \mathrm{O}$ exchangeable), $4.55(\mathrm{~d}, 4 \mathrm{H}, J=8.8 \mathrm{~Hz}, 4 \mathrm{NHCH}, \mathrm{L}-\mathrm{Ile}), 4.35-4.33(\mathrm{~m}, 1 \mathrm{H}, \mathrm{CH}, \mathrm{L}-\mathrm{Lys}), 4.20(\mathrm{~s}, 2 \mathrm{H}$, $\mathrm{NH}_{2}$ ), 3.70-3.65 (m, 2H, NHCH ${ }_{2}$, L-Lys), 2.55-2.40 (m, 4H, 4NHCHCH, L-Ile), 2.10-1.65 (m, 6H, 3CH $\left.\mathrm{NHCH}_{2} \mathrm{CH}_{2} \mathrm{CH}_{2} \mathrm{CH}_{2} \mathrm{CHNH}\right), 1.40-1.25$ (m, 8H, 4CH, L-Ile), $1.15-0.75$ (m, 24H, $8 \mathrm{CH}_{3}$, L-Ile). MS (EI, $70 \mathrm{eV}): m / z(\%)=743(\mathrm{M}, 1.39), 709$ (2), 656 (60.81), 616 (94.4), 584 (70.53), 543 (27.33), 515 (20.75), 471 (100), 443 (67.27), 358 (55.32), 302 (80.56), 86 (80.07), 69 (42.70), 57 (15.46), 50 (1.24). Analysis for $\mathrm{C}_{37} \mathrm{H}_{61} \mathrm{~N}_{9} \mathrm{O}_{7}$ (743.90): Calcd. C, 59.74; H, 8.26; N, 16.95. Found: C 59.71, H 8.17, N 16.92.

Cyclo-(N ${ }^{\alpha}$-dipicolinoyl)-bis[L-ILe-L-Phe]-L-Lys-NHNH 2 (6b). Yield: $65 \%$; m.p. $161-163{ }^{\circ} \mathrm{C},[\alpha]_{\mathrm{D}}^{25}:-40(\mathrm{C}$, 0.02, $\mathrm{MeOH})$. IR $\left(\mathrm{KBr}, \mathrm{cm}^{-1}\right): v=3289(\mathrm{NH}$ stretching), $3063(\mathrm{CH}$, aromatic), $2964(\mathrm{CH}$, aliphatic), 1650, 1529, 1448 and 1382 (C=O amide I, II, III and IV, respectively). ${ }^{1} \mathrm{H}-\mathrm{NMR}$ (500 MHz, ppm, DMSO- $d_{6}$ ): $\delta$ $=9.12\left(\mathrm{~s}, 1 \mathrm{H}, \mathrm{CONH}, \mathrm{D}_{2} \mathrm{O}\right.$ exchangeable, Hydrazide), 8.53-8.51 (m, 3H, Pyr-H), 8.24, 8.19 $(2 \mathrm{~s}, 6 \mathrm{H}, 6 \mathrm{NH}$, $\mathrm{D}_{2} \mathrm{O}$ exchangeable), 7.23-7.05 (m, 10H, Ar-H, L-Phe-ala), 4.50-4.48 (m, 6H, $2 \mathrm{NHCH}, 2 \mathrm{CH}_{2}$, L-Phe-ala), $4.45-4.38(\mathrm{~m}, 3 \mathrm{H}, 3 \mathrm{CH}), 4.35\left(\mathrm{~s}, 2 \mathrm{H}, \mathrm{NH}_{2}\right), 3.32\left(\mathrm{t}, 2 \mathrm{H}, 2 \mathrm{NHCH}_{2}, \mathrm{~L}-\mathrm{Lys}\right), 2.79-2.75(\mathrm{~m}, 2 \mathrm{H}, 2 \mathrm{NHCHCH}$, L-Ile), 1.35-1.34 (m, 6H, 3CH $\left.\mathrm{CH}_{2} \mathrm{NHCH}_{2} \mathrm{CH}_{2} \mathrm{CH}_{2} \mathrm{CH}_{2} \mathrm{CHNH}\right), 1.23-1.20$ (m, 4H, 2CH $\mathrm{CH}_{2}$ L-Ile), 0.90-0.60 $\left(\mathrm{m}, 12 \mathrm{H}, 4 \mathrm{CH}_{3}, \mathrm{~L}-\mathrm{Ile}\right) .{ }^{13} \mathrm{C}-\mathrm{NMR}\left(125 \mathrm{MHz}, \mathrm{ppm}, \mathrm{DMSO}-d_{6}\right): \delta=173.4,173.0$ (4C, 4CO, L-Phe-ala, L-Ile), 170.0 (1C, CO , Hydrazide), 164.5, 163.8 (2CO, Pyridine dicarbonyl), 150.7 (2C, pyridine, $\mathrm{C}_{2}, \mathrm{C}_{6}$ ), 142.9 (1C, pyridine, $\mathrm{C}_{4}$ ), 128.1 (2 C, pyridine, $\left.\mathrm{C}_{3}, \mathrm{C}_{5}\right), 139.0,127.8,126.7,125.5$ (12C, Ph-C), 60.6, 54.0

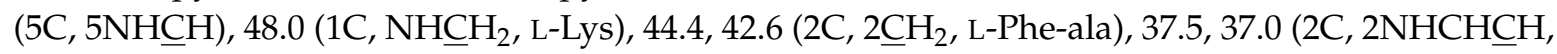
L-Ile), 34.0, $30.9\left(5 \mathrm{C}, 5 \mathrm{CH}_{2}\right), 24.2,10.5\left(4 \mathrm{C}, 4 \mathrm{CH}_{3}, \mathrm{~L}-\mathrm{Ile}\right) . \mathrm{MS}(\mathrm{EI}, 70 \mathrm{eV}): m / z(\%)=813\left(\mathrm{M}^{+}+1,2.42\right)$, 724 (13.09), 684 (40.82), 669 (30.09), 549 (37.88), 477 (55.84), 302 (100), 84 (24.44), 55 (22.15), 50 (3.04). Analysis for $\mathrm{C}_{43} \mathrm{H}_{57} \mathrm{~N}_{9} \mathrm{O}_{7}$ (812.00): Calcd. C, 63.61; H, 7.08; N, 15.53. Found: C 63.59, H 7.02, N 15.47. 
Cyclo-(N ${ }^{\alpha}$-Dipicolinoyl)-bis[L-Phe-L-ILe]-L-Lys-NHNH 2 (6c). Yield: 78\%; m.p. 208-210 ${ }^{\circ} \mathrm{C},[\alpha]_{\mathrm{D}}^{25}:-66(\mathrm{C}$, 0.02, MeOH). IR (KBr, cm $\left.{ }^{-1}\right): v=3282$ (NH stretching), 3065 (CH, aromatic), 2963 (CH, aliphatic), 1645, 1524, 1447 and 1382 (C=O amide I, II, III and IV, respectively). ${ }^{1} \mathrm{H}-\mathrm{NMR}\left(500 \mathrm{MHz}, \mathrm{ppm}, \mathrm{DMSO}-d_{6}\right)$ : $\delta=9.35$ (s, 1H, CONH, $\mathrm{D}_{2} \mathrm{O}$ exchangeable, Hydrazide), 9.10-8.90 (m, 3H, Pyr-H), 8.11, 8.09 (2s, 6H, $6 \mathrm{NH}, \mathrm{D}_{2} \mathrm{O}$ exchangeable), 7.41-7.13 (m, 10H, Ar-H, L-Phe-ala), 4.82-4.80 (m, 6H, 2NHCH, $2 \mathrm{CH}_{2}$, L-Phe-ala), 4.25-4.00 (m, 3H, 3CH), 3.98 (s, 2H, NH 2$), 3.30-3.19$ (m, 2H, NHCH 2 , L-Lys), 2.75-2.70 (m, 2H, 2NHCHCH, L-Ile), 1.4-1.32 (m, 6H, $\left.\mathrm{NHCH}_{2} \mathrm{CH}_{2} \mathrm{CH}_{2} \mathrm{CH}_{2} \mathrm{CHNH}\right), 1.14-1.12$ (m, 4H, 2CH L-Ile), 0.80-0.79 (m, 12H, 4CH 3 , L-Ile). ${ }^{13} \mathrm{C}-\mathrm{NMR}\left(125 \mathrm{MHz}, \mathrm{ppm}, \mathrm{DMSO}-d_{6}\right): \delta=174.6,174.0(4 \mathrm{C}, 4 \mathrm{CO}$, L-Phe-ala, L-Ile), 172.0 (1C, CO, Hydrazide), 168.5, 166.9 (2C, 2CO, Pyridine dicarbonyl), 148.5 (2C, pyridine, $\mathrm{C}_{2}, \mathrm{C}_{6}$ ), 144.0 (1C, pyridine, $\left.\mathrm{C}_{4}\right), 126.1$ (2C, pyridine, $\left.\mathrm{C}_{3}, \mathrm{C}_{5}\right), 138.5,127.0,126.7,125.8(12 \mathrm{C}$, Ph-C), 61.0, 57.5 (5C, 5NHCH ), 50.0 (1C, $\mathrm{NHCH}_{2}$, L-Lys), 47.4, 46.9 (2C, 2 $\underline{C H}_{2}$, L-Phe-ala), 39.0, 38.8

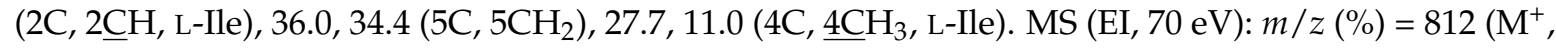
0.8), 808 (2.6), 621 (1.00), 423 (1.2), 391 (1.2), 373 (1.8), 295 (3.6), 268 (11.9), 213 (11.3), 149 (17.2), 121 (26.9), 104 (36.2), 91 (100), 86 (43.4), 77 (35.8), 50 (24.2). Analysis for $\mathrm{C}_{43} \mathrm{H}_{57} \mathrm{~N}_{9} \mathrm{O}_{7}$ (812.00): Calcd. C, 63.61; H, 7.08; N, 15.53. Found: C 63.58, H 7.07, N 15.49.

\subsection{Anticancer Screening}

In vitro bioassay on human cancer cell lines was adopted against two cancer cell lines (MCF-7 and HepG-2). HepG2, human hepatocellular carcinoma and MCF-7, human breast cancer cell lines were obtained from Sigma-Aldrich Chemie GmbH, Taufkirchen, Germany. Tamoxifen and 5-fluorouracil were used as reference standards according to a previously reported method [35-39].

\subsection{In Vitro Enzymatic Assays}

The in vitro enzyme inhibition determination for compound $5 \mathrm{c}$ was carried out in confirmatory diagnostic unit, Vacsera, Egypt. The evaluation performed profiling of the compound $\mathbf{5 c}$ against a range of four protein kinases [VEGFR-2, EGFR, PDGFR $\beta$ and CDK-2] by ELISA assay method using staurosporine as a reference according to the previously reported methods [40-42].

\subsection{Molecular Modeling Studies}

To evaluate the inhibitory activity and confirm best interactions between compound $5 \mathrm{c}$ and target proteins, molecular docking studies were performed with the help of Molecular Operating Environment $\left(\mathrm{MOE}^{\circledR}\right)$ 2008.10 [30,31]. The three-dimensional X-ray structures of VEGFR-2 (PDB code: 4ASD) [33] and CDK-2 (PDB code: 2J9M) [34] were obtained from the Protein Data Bank through the internet.

\section{Conclusions}

In summary, a series of macrocyclic derivatives bearing pyrido-pentapeptide moiety were designed and synthesized from $N, N$-bis-[1-carboxy-2-(benzyl)]-2,6-(diaminocarbonyl)pyridine. Two human cancer cell lines (MCF-7 and HepG-2) were used to evaluate the anticancer potency of all synthesized compounds. Compared with tamoxifen, compounds $\mathbf{4 a}, \mathbf{4 b}, \mathbf{5 a}, \mathbf{5 b}, \mathbf{5 c}, \mathbf{6 a}, \mathbf{6 b}, \mathbf{6 c}$ exhibited excellent potency against one or both cell lines. Furthermore, compound $5 \mathrm{c}$ showed the strongest cytotoxic activities against both cell lines $\left(\mathrm{IC}_{50}=9.41 \pm 1.25\right.$ and $7.53 \pm 1.33 \mu \mathrm{M}$, respectively) in comparison with tamoxifen and 5-fluorouracil. On the other hand, enzyme inhibition assay of compound 5c against VEGFR-2, CDK-2 and PDGFR $\beta$ demonstrated promising inhibitory activity with $\mathrm{IC}_{50} 0.01 \pm 1.25,0.06 \pm 1.27$ and $0.08 \pm 1.45 \mu \mathrm{M}$, respectively compared to staurosporine. Molecular docking study showed the ability of the compound to fit into the active sites of these enzymes with the best binding modes. It is worthy to mention macrocyclic pyrido-pentapeptide based compounds serve as a useful template for the further development of the anticancer multi-targeted agents. 
Author Contributions: The listed authors contributed to this work as described in the following. A.E.-G.E.A., M.H.A.-G., G.O.M. gave the concepts of the work, interpreted the results, the experimental part and prepared the manuscript, M.A.A.-O. cooperated in the preparation of the manuscript and E.S.N. and E.A.E. performed the anticancer and biochemical, as well as docking studies of the new compounds. All authors read and approved the final manuscript.

Funding: This research was funded by research group project "RGP-1435-047" of the Deanship of Scientific Research, King Saud University.

Acknowledgments: The authors are grateful to the Deanship of Scientific Research, King Saud University for funding this work through research group project "RGP-1435-047".

Conflicts of Interest: The authors declare no conflict of interest.

\section{References}

1. Dissanayake, S.; Denny, W.A.; Gamage, S.; Sarojini, V. Recent developments in anticancer drug delivery using cell penetrating and tumor targeting peptides. J. Controll. Release 2017, 250, 62-76. [CrossRef] [PubMed]

2. Driggers, E.M.; Hale, S.P.; Lee, J.; Terrett, N.K. The exploration of macrocycles for drug discovery-An underexploited structural class. Nat. Rev. Drug Discov. 2008, 7, 608-624. [CrossRef] [PubMed]

3. Krahn, D.; Ottmann, C.; Kaiser, M. Macrocyclic proteasome inhibitors. Curr. Med. Chem. 2011, 18, $5052-5060$. [CrossRef] [PubMed]

4. Marsault, E.; Peterson, M.L. Macrocycles Are Great Cycles: Applications, Opportunities, and Challenges of Synthetic Macrocycles in Drug Discovery. J. Med. Chem. 2011, 54, 1961-2004. [CrossRef] [PubMed]

5. Erb, W.; Zhu, J. From natural product to marketed drug: The tiacumicin odyssey. Nat. Prod. Rep. 2013, 30, 161-174. [CrossRef] [PubMed]

6. Mallinson, J.; Collins, I. Macrocycles in new drug discovery. Future Med. Chem. 2012, 4, 1409-1438. [CrossRef] [PubMed]

7. Felício, M.R.; Silva, O.N.; Gonçalves, S.; Santos, N.C.; Franco, O.L. Peptides with dual antimicrobial and anticancer activities. Front. Chem. 2017, 5, 5. [CrossRef] [PubMed]

8. Amr, A.E.; Abo-Ghaliaa, M.H.; Abdalah, M.M. Synthesis of novel macrocyclic peptido-calix[4]arenes and peptidopyridines as precursors for potential molecular metallacages, chemosensors and biologically active candidates. Z. Naturforsch. 2006, 61b, 1335-1345. [CrossRef]

9. Amr, A.E.; Abdel-Salam, O.I.; Attia, A.; Stibor, I. Synthesis of new potential bis-intercallators based on chiral pyridine-2,6-dicarbox-amides. Collect. Czech Chem. Commun. 1999, 64, 288-298. [CrossRef]

10. Attia, A.; Abdel-Salam, O.I.; Amr, A.E.; Stibor, I.; Budesinsky, M. Synthesis and antimicrobial activity of some new chiral bridged macrocyclic pyridines. Egypt. J. Chem. 2000, 43, 187-201. [CrossRef]

11. Naglah, A.M.; Moustafa, G.O.; Al-Omar, M.A.; Al-Salem, H.S.A.; Hozzein, W.N. Synthesis, characterization and in vitro antimicrobial investigation of novel amino acids and dipeptides based on dibenzofuran-2-sulfonyl-chloride. J. Comput. Theor. Nanosci. 2017, 14, 3183-3190. [CrossRef]

12. Al-Omar, M.A.; Amr, A.E. Synthesis of some new pyridine-2,6-carboxamide-derived Schiff Bases as potential antimicrobial agents. Molecules 2010, 15, 4711-4721. [CrossRef] [PubMed]

13. Abd El-Salam, O.I.; Al-Omar, M.A.; Fayed, A.A.; Flefel, E.M.; Amr, A.E. Synthesis of new macrocyclic polyamides as antimicrobial agent candidates. Molecules 2012, 17, 14510-14521. [CrossRef] [PubMed]

14. Al-Salem, H.S.A.; Naglah, A.M.; Moustafa, G.O.; Mahmoud, A.Z.; Al-Omar, M.A. Synthesis of novel tripeptides based on dibenzofuran-2-sulfonyl-[aromatic and hydroxy aromatic residues]: Towards antimicrobial and antifungal agents. J. Comput. Theor. Nanosci. 2017, 14, 3958-3966. [CrossRef]

15. Moustafa, G.; Khalaf, H.; Naglah, A.; Al-Wasidi, A.; Al-Jafshar, N.; Awad, H. Synthesis, molecular docking studies, in vitro antimicrobialand antifungal activities of novel dipeptide derivatives based on n-(2-(2-hydrazinyl-2-oxoethylamino)-2-oxoethyl)-nicotinamide. Molecules 2018, 23, 761. [CrossRef] [PubMed]

16. Khayyat, S.; Amr, A.E. Synthesis and biological activities of some new ( $\mathrm{N}^{\alpha}$-dinicotinoyl)-bis-L- leucyl lnear and macrocyclic peptides. Molecules 2014, 19, 10698-10716. [CrossRef] [PubMed]

17. Amr, A.E.; Abo-Ghalia, M.H.; Abdalah, M.M. Synthesis of new ( ${ }^{\alpha}{ }^{\alpha}$ dipicolinoyl)-bis-L-valyl-L-phenyl linear and macrocyclic bridged peptides as anti-inflammatory agents. Arch. Pharm. Chem. Life Sci. 2007, 340, 304-309. [CrossRef] [PubMed]

18. Abo-Ghalia, M.H.; Amr, A.E. Synthesis and investigation of a new cyclo $\left(\mathrm{N}^{\alpha}\right.$-pentapeptide of a breast and CNS cytotoxic activity and an ionophoric specificity. Amino Acids 2004, 26, 283-289. [CrossRef] [PubMed] 
19. Masereel, B.; Dupont, L.; Laeckmann, D.; Liégeois, J.F.; Pirotte, B.; de Tullio, P.; Delarge, J. Synthesis and pharmacology of pyrid-3-ylsulfonylcyanoguanidines as diuretic. Eur. J. Med. Chem. 1995, 30, 235-240. [CrossRef]

20. Abo-Ghalia, M.H.; Moustafa, G.O.; Alwasidi, A.S.; Naglah, A.M. Cytotoxic investigation of isophthaloyl cyclopentapeptides. Lat. Am. J. Pharm. 2017, 36, 1957-1962. [CrossRef]

21. Moustafa, G.O.; El-Sawy, A.A.; Abo-Ghalia, M.H. Synthesis of novel cyclopeptide candidates: I-cyclo-[N ${ }^{\alpha}$-isophthaloyl-bis-(Glycine-amino acid)-L-lysine] derivatives with expected anticancer activity. Egypt. J. Chem. 2013, 5, 473-494. [CrossRef]

22. Amr, A.E.; Mohamed, A.M.; Ibrahim, A.A. Synthesis of some new chiral tricyclic and macrocyclic pyridine derivatives as antimicrobial agents. Z. Naturforsch. 2003, 58b, 861-868. [CrossRef]

23. Abo-Ghaliaa, M.H.; Amr, A.E.; Abdalah, M.M. Synthesis of some new ( $\mathrm{N}^{\alpha}$-dipicolinoyl)-bisL-leucyl-DL-norvalyl linear tetra and cyclic octa bridged peptides as new antiinflammatory agents. Z. Naturforsch. 2003, 58b, 903-910. [CrossRef]

24. Patrick, G.L. An Introduction to Medicinal Chemistry, 3rd ed.; Oxford University Press Inc.: New York, NY, USA, 2005; pp. 489-553. ISBN 9780198749691.

25. Hu, S.; Yu, H.; Zhao, L.; Liang, A.; Liu, L.; Zhang, H. Molecular docking and 3D-QSAR studies on checkpoint kinase 1 inhibitors. Med. Chem. Res. 2013, 22, 4992-5013. [CrossRef]

26. Ali, S.; Singh, V.; Jain, P.; Tripathi, V. Synthesis, antibacterial, anticancer and molecular docking studies of macrocyclic metal complexes of dihydrazide and diketone. J. Saudi Chem. Soc. 2018. [CrossRef]

27. Mariaule, G.; Belmont, P. Cyclin-Dependent Kinase Inhibitors as Marketed Anticancer Drugs: Where Are We Now? A Short Survey. Molecules 2014, 19, 14366-14382. [CrossRef] [PubMed]

28. Sharma, S.; Singh, J.; Ojha, R.; Singh, H.; Kaur, M.; Bedi, P.; Nepali, K. Design Strategies, Structure Activity Relationship and Mechanistic Insights for Purines as Kinase Inhibitors. Eur. J. Med. Chem. 2016, 112, $298-346$. [CrossRef] [PubMed]

29. Abo-Ghalia, M.H.; Abd El-Hamid, M.; Zweil, M.A.; Amr, A.E.; Moafi, S.A. Synthesis and reactions of new chiral linear and macrocyclic tetra- and penta-peptide candidates. Z. Naturforsch. B 2012, 67, 806-818. [CrossRef]

30. Molecular Operating Environment (MOE), 2008.10. Available online: https://www.chemcomp.com/MOEMolecular_Operating_Environment.htm (accessed on 12 June 2018).

31. Chemical Computing Group ULC. Available online: https:/ / www.bloomberg.com/profiles/companies / 1522230D:CN-chemical-computing-group-ulc (accessed on 23 June 2018).

32. Conconi, M.T.; Marzaro, G.; Urbani, L.; Zanusso, I.; Di Liddo, R.; Castagliuolo, I.; Brun, P.; Tonus, F.; Ferrarese, A.; Guiotto, A.; et al. Quinazoline-based multi-tyrosine kinase inhibitors: Synthesis, modeling, antitumor and antiangiogenic properties. Eur. J. Med. Chem. 2013, 67, 373-383. [CrossRef] [PubMed]

33. Mctigue, M.; Murray, B.W.; Chen, J.H.; Deng, Y.; Solowiej, J.; Kania, R.S. Molecular Conformations, Interactions, and Properties Associated with Drug Efficiency and Clinical Performance Among Vegfr Tk Inhibitors. Proc. Natl. Acad. Sci. USA 2012, 109, 18281-18289. [CrossRef] [PubMed]

34. Luecking, U.; Siemeister, G.; Schaefer, M.; Briem, H.; Krueger, M.; Lienau, P.; Jautelat, R. Macrocyclic Aminopyrimidines as Multitarget Cdk and Vegf-R Inhibitors with Potent Antiproliferative Activities. Chem. Med. Chem. 2007, 2, 63-77. [CrossRef] [PubMed]

35. Al-Salahi, R.; Elsayed, E.A.; El Dib, R.A.; Wadaan, M.; Ezzeldin, E.; Marzouk, M. Synthesis, characterization and cytotoxicity evaluation of 5-hydrazono-[1,2,4]triazolo[1,5-a]quinazolines (Part I). Lat. Am. J. Pharm. 2016, 35, 58-65.

36. Al-Salahi, R.; Elsayed, E.A.; El Dib, R.A.; Wadaan, M.; Ezzeldin, E.; Marzouk, M. Cytotoxicity of new 5-hydrazono-[1,2,4]triazolo[1,5-a]quinazolines (Part II). Lat. Am. J. Pharm. 2016, 35, 66-73.

37. Elsayed, E.A.; Sharaf-Eldin, M.A.; Wadaan, M. In vitro evaluation of cytotoxic activities of essential oil from Moringa oleifera seeds on HeLa, HepG2, MCF-7, CACO-2 and L929 cell lines. Asian Pac. J. Cancer Preven. 2015, 16, 4671-4675. [CrossRef] [PubMed]

38. Elsayed, E.A.; Farooq, M.; Dailin, D.; El-Enshasy, H.A.; Othman, N.Z.; Malek, R.; Danial, E.; Wadaan, M. In vitro and in vivo biological screening of kefiran polysaccharide produced by Lactobacillus kefiranofaciens. Biomed. Res. 2017, 28, 594-600.

39. Vichai, V.; Kirtikara, K. Sulforhodamine B colorimetric assay for cytotoxicity screening. Nat. Protoc. 2006, 1, 1112-1116. [CrossRef] [PubMed] 
40. El-Husseiny, W.M.; El-Sayeda, M.A.-A.; Abdel-Aziz, N.I.; El-Azab, A.S.; Ahmed, E.R.; Abdel-Aziz, A.A.-M. Synthesis, antitumour and antioxidant activities of novel a,b-unsaturated ketones and related heterocyclic analogues: EGFR inhibition and molecular modeling study. J. Enzyme Inhib. Med. Chem. 2018, 33, 507-518. [CrossRef] [PubMed]

41. Mouineer, A.A.; Zaher, A.F.; El Malah, A.A.; Sobh, E.A.E. Design, synthesis, antitumor activity, cell cycle analysis and ELISA assay for cdk-2 of a new (4-aryl-6-flouro-4h-benzo [4,5] thieno [3,2- $b]$ pyran) derivatives. J. Chem. Pharm. Res. 2017, 9, 106-120.

42. Abdullaziz, M.A.; Abdel-Mohsen, H.T.; El Kerdawy, A.M.; Ragab, F.A.F.; Ali, M.M.; Abu-bakr, S.M.; Girgis, A.S.; El Diwani, H.I. Design, synthesis, molecular docking and cytotoxic evaluation of novel 2-furybenzimidazoles as VEGFR-2 inhibitors. Eur. J. Med. Chem. 2017, 136, 315-329. [CrossRef] [PubMed]

Sample Availability: Samples of the compounds are available from the authors.

(C) 2018 by the authors. Licensee MDPI, Basel, Switzerland. This article is an open access article distributed under the terms and conditions of the Creative Commons Attribution (CC BY) license (http:/ / creativecommons.org/licenses/by/4.0/). 